NASA Contractor Report 4519

\title{
An Analysis for High Reynolds Number Inviscid/Viscid Interactions in Cascades
}

Mark Barnett, Joseph M. Verdon, and Timothy C. Ayer

United Technologies Research Center

East Hartford, Connecticut

May 1993

Prepared for the

Lewis Research Center

Under Contract NAS3-25425 


\section{An Analysis for High Reynolds Number Inviscid/Viscid Interactions in Cascades}

\section{Contents}

$\begin{array}{ll}\text { Summary } & 1\end{array}$

1 Introduction $\quad 2$

2 General Concepts $\quad 4$

3 Inviscid Region $\quad 6$

4 Viscous Layer $\quad 8$

5 Inviscid/Viscid Interaction $\quad 12$

6 Numerical Examples $\quad 13$

6.1 Compressor Exit Guide Vane . . . . . . . . . . . . . . . . . . . . 14

6.2 High-Speed Compressor . . . . . . . . . . . . . . . . . . 15

6.3 Turbine .............................. 16

6.4 Timing Study and Convergence Behavior . . . . . . . . . . . . . . . 17

7 Concluding Remarks $\quad 19$

$\begin{array}{lr}\text { References } & 20\end{array}$

$\begin{array}{ll}\text { List of Symbols } & 23\end{array}$

$\begin{array}{lr}\text { List of Figures } & 26\end{array}$

$\begin{array}{lr}\text { Figures } 1 \text { through } 13 & \mathbf{2 7}\end{array}$

A Details of the Viscous-Layer Solution Procedure $\quad 40$

A.1 Finite-Difference Approximations . . . . . . . . . . . . . . . . . . 40

A.2 Surface Procedure . . . . . . . . . . . . . . . . . . . . . . . . . . . . . . . . . . . . . . . . . . . . . . . . . . . .

A.3 Wake Procedure . . . . . . . . . . . . . . . . . . . . . . . 44 


\title{
AN ANALYSIS FOR HIGH REYNOLDS NUMBER INVISCID/VISCID INTERACTIONS IN CASCADES*
}

\author{
Mark Barnett, Joseph M. Verdon, and Timothy C. Ayer \\ United Technologies Research Center \\ East Hardford, Connecticut
}

\section{Summary}

An efficient steady analysis for predicting strong inviscid/viscid interaction phenomena such as viscous-layer separation, shock/boundary-layer interaction and trailing-edge/nearwake interaction in turbomachinery blade passages is needed as part of a comprehensive analytical blade design prediction system. Such an analysis is described in the present report. It uses an inviscid/viscid interaction approach, in which the flow in the outer inviscid region is assumed to be potential, and that in the inner or viscous-layer region is governed by Prandtl's equations. The inviscid solution is determined using an implicit, least-squares, finite-difference approximation, the viscous-layer solution using an inverse, finite-difference, space-marching method which is applied along the blade surfaces and wake streamlines. The inviscid and viscid solutions are coupled using a semi-inverse global iteration procedure, which permits the prediction of boundary-layer separation and other strong-interaction phenomena. Results are presented for three cascades, with a range of inlet flow conditions considered for one of them, including conditions leading to large-scale flow separations. Comparisons with Navier-Stokes solutions and experimental data are also given. 


\section{Introduction}

An important problem faced by engine designers is the prediction of high Reynolds number $(\mathrm{Re})$ viscous flow and, in particular, viscous separation phenomena in compressor and turbine blade passages. Viscous effects control aerodynamic losses, heat transfer rates and stall, and hence, must be accounted for. In addition to steady-flow applications, the ability to account for viscous effects in unsteady flows is needed for aeroelastic and aeroacoustic design predictions, e.g., to predict the onset of stall flutter, blade row interactions due to the convection of viscous wakes from upstream rows and other unsteady effects which impact the structural and acoustic characteristics of turbomachinery blade rows. Clearly, efficient analytical procedures for predicting steady and unsteady viscous flows in high-performance compressor and turbine blading would be a significant contribution to a successful blade design prediction system.

The analysis described in this report is being developed as part of a research program which has the goal of constructing reliable and efficient theoretical prediction methods for steady and unsteady viscous flows, at high Reynolds numbers, through two- and quasithree-dimensional subsonic and transonic cascades. The approach to be followed is similar to that which has been applied successfully in external aerodynamics, where inviscid/viscid interaction (IVI) concepts have been used to predict the complete steady (e.g., Refs. [1] and [2]), and unsteady (e.g., Refs. [3] and [4]) flow fields.

Thus, for example, for high Reynolds number flow through a cascade, the complete flow can be divided conceptually into two regions: an "outer" inviscid region and an "inner" viscous region. The construction of a general viscous cascade solver involves first, the development of component flow solvers, and second, the implementation of these component solvers into an overall computational procedure to produce a complete analysis. Solution methods for steady subsonic and transonic inviscid flows through cascades (e.g., Ref. [5]) and for steady boundary-layer and wake flows (e.g., Refs. [6] and [7]) have been developed to a relatively mature state. Methods for coupling such solutions have also been developed and assessed through a number of model problem studies (e.g., Refs. [6]-[8]). Inviscid/viscid interaction procedures for predicting steady flow in cascades have been developed (e.g., Refs. [9]-[13]) and applied over a wide range of inlet flow conditions, including conditions leading to stall [13]. A similar approach can be developed for unsteady flows; for example, a linearized inviscid analysis [14] and a weak-interaction unsteady viscous-layer analysis [15] have been coupled and applied to predict attached flows [16].

The focus of this report is on the development of an accurate and efficient steady cascade analysis that will provide the foundation for an unsteady procedure to be developed later. The ability of a steady inviscid/viscid interaction analysis to predict compressor cascade performance with accuracy comparable to that of a state-of-the-art Navier-Stokes (N-S) analysis has been demonstrated in Ref. [13]. Here, an Euler inviscid calculation was coupled to a finite-difference viscous-layer analysis. Although accurate, this approach, like current $\mathrm{N}-\mathrm{S}$ analyses, requires too much computational effort for repetitive design calculations. The present effort is intended to realize the additional goal, beyond that of accuracy, of providing an efficient analysis suitable for design applications.

In the present approach, the "outer" inviscid flow is determined using potential-flow the- 
ory; thus, it is assumed that the inviscid flow is isentropic and irrotational. The steady, cascade, full-potential analysis (SFLOW) of Hoyniak and Verdon [17] is employed. SFLOW was constructed for use with the LINearized inviscid unsteady FLOw (LINFLO) analysis of Verdon and Caspar [14], to provide a comprehensive and compatible steady and unsteady inviscid flow prediction capability for cascades. In the present calculation procedure (which will be referred to as SFLOW-IVI), viscous effects are incorporated by adjusting the blade and wake surface boundary conditions in SFLOW to account for the effects of viscous displacement thickness. The nonlinear inviscid analysis, coupled with the IVI iteration procedure, allows nonlinear changes to the base flow due to viscous effects to be evaluated. The ability to treat nonlinear perturbations is especially important in transonic flows in which shock positions are significantly altered by viscous displacement effects. Although the analysis described in this report is presently restricted to subsonic flows, it will be extended to treat transonic flows in the future. 


\section{General Concepts}

For flows of practical interest in either external or internal aerodynamics, the Reynolds number is usually sufficiently high so that the flow past an airfoil or blade can be divided into two regions: an "inner" dissipative region consisting of boundary layers and wakes, and an "outer" inviscid region. The principal interaction between the flows in the viscid and inviscid regions arises from the displacement thickness effect which leads to thickened semiinfinite equivalent bodies with corresponding changes in surface pressures. If the interaction is "weak," then the complete flow problem can be solved sequentially. This traditional approach for calculating the interaction between the inviscid and viscous parts of the flow is based on a direct hierarchy between the two regions, which is applicable as long as the disturbances to the inviscid flow due to the viscous displacement effect remain weak.

Flows over airfoils, however, involve both a weak overall interaction arising from standard displacement thickness and wake curvature effects, and local strong-displacement interactions caused, for example, by viscous-layer separations, shock/boundary-layer interactions, and trailing-edge/near-wake interactions. These features can lead to singularities in a classical boundary-layer solution and a subsequent breakdown of a weak-interaction solution procedure. In addition, viscous displacements in the strong-interaction region cause substantial changes in the local inviscid pressure field and can, in some cases (e.g., in flows with large-scale separations), cause substantial changes in the global pressure field as well. The concept of an inner viscous region and an outer inviscid region still holds, but the classical hierarchical structure of the flow no longer applies. Thus, in a local strong-interaction region, the hierarchy changes from "direct" (i.e., pressure determined by the inviscid flow) to "interactive" (i.e., pressure determined by a mutual interaction between the inviscid and the viscous-layer flows), and this change must be accommodated within a comprehensive inviscid/viscid interaction analysis.

In a classical weak-interaction calculation, the blade surface and wake streamline pressure distribution is determined from the zeroth-order inviscid solution, that is, the inviscid solution unperturbed by viscous effects. This distribution is imposed when solving the viscous-layer equations using the direct approach mentioned above. The resulting displacement thickness distribution, $\delta(s)$, is then used to obtain the first-order (perturbation) inviscid solution, thereby accounting for the changes to the base inviscid flow due to viscous displacement effects. The resulting changes in the blade pressure distributions and in the downstream freestream flow properties (e.g., Mach number and flow angle) can then be calculated. It is sometimes possible to continue this sequential solution procedure until a converged solution for the entire flow is achieved. This hierarchical approach, known as Prandtl iteration, works well if the interaction between the flows in the viscid and inviscid regions is weak. However, local regions of strong inviscid/viscid interaction are inevitably present in realistic airfoil flows. When the strong interaction leads to viscous-layer separation the Prandtl iteration usually fails and an alternative approach must be used.

It is well known that the difficulties associated with the Prandtl iteration stem from the specification of the pressure in the viscous layer. If the boundary layer separates, the Goldstein singularity [18] prevents a continuation of the direct viscous-layer solution downstream of the predicted separation point [19]. This difficulty can be circumvented by using 
an inverse viscous-layer solution procedure in which the displacement thickness is specified instead of the pressure. The inverse procedure permits viscous solutions to be continued through local strong-interaction regions, including separated flow regions.

The approach taken here employs an IVI model to calculate high Reynolds number $(R e)$ flows through two-dimensional cascades. The flow in the outer inviscid region is governed by the full-potential equation and that in the inner viscous region by Prandtl's viscouslayer equations. The non-hierarchical nature of strong interactions is accounted for in the procedure used to couple the two solutions.

We consider high Reynolds number $\left(R e=\rho_{-\infty}^{*} V_{-\infty}^{*} L^{*} / \mu_{-\infty}^{*}\right)$ steady flow, with negligible body forces, of a perfect gas with constant specific heats and Prandtl number through a two-dimensional cascade, as shown in Fig. 1. In the following discussion, all flow variables and spatial coordinates are dimensionless. Lengths have been scaled with respect to the blade chord $\left(L^{*}\right)$, density, velocity and viscosity with respect to their inlet freestream values $\left(\rho_{-\infty}^{*}, V_{-\infty}^{*}\right.$ and $\mu_{-\infty}^{*}$, respectively), pressure with respect to twice the inlet freestream dynamic pressure $\left(\rho_{-\infty}^{*} V_{-\infty}^{* 2}\right)$, and temperature with respect to the square of the inlet freestream speed divided by the specific heat at constant pressure $\left(V_{-\infty}^{* 2} / c_{p}^{*}\right)$. Here the superscript $*$ denotes a dimensional quantity and the subscript $-\infty$ refers to the prescribed freestream conditions far upstream. 


\section{Inviscid Region}

Since the inviscid flow is assumed to be isentropic and irrotational, a velocity potential, $\Phi$, exists and is governed by the field equation

$$
A^{2} \nabla^{2} \Phi=\nabla \Phi \cdot \nabla(\nabla \Phi)^{2} / 2
$$

The speed of sound propagation, $A$, the fluid pressure, $P$, density, $\rho$, and temperature, $T$, are related through Bernoulli's equation and the isentropic relations as follows:

$$
\left(M_{-\infty} A\right)^{2}=\left(\gamma M_{-\infty}^{2} P\right)^{(\gamma-1) / \gamma}=\rho^{(\gamma-1)}=(\gamma-1) M_{-\infty}^{2} T=1-\frac{(\gamma-1)}{2} M_{-\infty}^{2}\left[(\nabla \Phi)^{2}-1\right],
$$

where $M$ is the Mach number and $\gamma$ is the specific heat ratio of the fluid. The inviscid flow is determined as a solution of Eq. (3.1) subject to a flow tangency condition at each blade surface, cascade periodicity conditions upstream and downstream of the blade row, jump conditions on normal velocity and pressure across blade wakes, and the appropriate uniform flow conditions far upstream of the blade row. Usually, a Kutta condition is applied at blade trailing edges in lieu of specifying the exit flow angle. Finally, far downstream of the blade row global mass conservation is enforced, accounting for blockage effects due to the viscous layers. For the flows considered here, the inlet and exit velocities are subsonic.

The specific forms of the blade and wake conditions follow from an asymptotic matching of the outer inviscid and the inner viscous-layer equations [1]. Thus, the inviscid solution for the normal velocity at a blade surface must match the viscous solution for this velocity at the outer edge of the viscous layer. It follows, after carrying out the asymptotic matching, that

$$
\left.\nabla \Phi \cdot \mathbf{n}\right|_{s}=\rho_{e}^{-1} d\left(\rho_{e} u_{e} \delta\right) / d s,
$$

where $\mathcal{S}$ denotes a reference blade surface (see Fig. 1), $\rho_{e}$ and $u_{e}$ are the inviscid density and velocity at this surface (or, the viscous density and streamwise velocity component at the edge of the viscous layer) and $\delta$ is the boundary-layer displacement thickness. The quantities $s$ and $\mathbf{n}$ denote the arc distance along the blade (positive in the downstream direction and zero at the leading-edge stagnation point) and the local unit normal vector directed outward from the surface, respectively.

Two types of terms arise from the wake matching conditions [1], one due to the displacement thickness effect of the wake and the other due to the wake curvature effect. The first leads to the requirement that the inviscid solution for the normal component of velocity must be discontinuous with jump given by

$$
\left.\llbracket \nabla \Phi \rrbracket \cdot \mathbf{n}\right|_{\mathcal{W}}=\rho_{e}^{-1} d\left(\rho_{e} u_{e} \delta_{W}\right) / d s,
$$

where 【』 denotes the difference in a quantity (upper minus lower) across the wake, $\mathbf{n}$ is the upward pointing unit normal vector to the reference wake streamline (i.e., $\mathcal{W}$ in Fig. 1 ), and $\delta_{W}$ is the displacement thickness of the complete wake. The wake curvature effect gives rise to a static pressure difference across the wake. The requirement that the outer inviscid flow match this pressure difference leads to the condition

$$
\llbracket P \rrbracket_{W}=\kappa \rho_{e} u_{e}^{2}\left(\delta_{W}+\theta_{W}\right),
$$


where $\theta_{W}$ is the momentum thickness of the complete wake and $\kappa$ is the curvature of the wake which is taken as positive when the reference wake streamline is concave upwards.

A complication arises in that the location of the reference wake streamline is unknown a priori; however, to within lowest order, the wake conditions can be referenced to any arbitrary curve emanating from the trailing edge and lying within the actual viscous wake [8]. In the present study, the reference wake streamline is taken to be the aft stagnation streamline as determined from the pure inviscid solution. This is adequate except in extreme cases where the location of the stagnation streamline is significantly altered by viscous effects. In this case, it is possible to periodically update the location of the wake streamline during the calculation, although this has not been done in the present study.

The boundary-value problem posed by Eqs. (3.1) and (3.2) is solved using Newton iteration. This is accomplished by substituting for the potential function $\Phi$ in Eqs. (3.1) and (3.2) using the expression

$$
\Phi=\Phi_{n}+\phi_{n},
$$

where $n$ represents the Newton iteration count, $\Phi_{n}$ is the initial guess or previous iteration value for the potential function at each point in the field and $\phi_{n}$ is the correction to $\Phi_{n}$. The variable $\phi_{n}$ is the quantity solved for during each Newton iteration. Higher-order terms (i.e., products of $\phi_{n}$ ) are dropped, resulting in a linear equation for $\phi_{n}$. This equation is discretized and the resulting matrix system of algebraic difference equations is inverted directly using lower-upper decomposition and Gaussian elimination. The Newton iterations are continued until $\left\|\phi_{n}\right\|<\varepsilon$, where $\varepsilon$ is a user specified convergence criterion $(\varepsilon \ll 1)$. The SFLOW analysis is described in detail in Ref. [17]. 


\section{Viscous Layer}

The flow in the inner or viscous region is assumed to be governed by Prandtl's viscouslayer equations. After introducing the scaled normal coordinate, $\tilde{n}$, and normal velocity component, $\tilde{v}$, where

$$
\tilde{n}=R e^{1 / 2} n \text { and } \tilde{v}=R e^{1 / 2} v,
$$

the continuity and streamwise momentum equations have the form

$$
\frac{\partial(\rho u)}{\partial s}+\frac{\partial(\rho \tilde{v})}{\partial \tilde{n}}=0
$$

and

$$
\rho\left[u \frac{\partial u}{\partial s}+\tilde{v} \frac{\partial \tilde{v}}{\partial \tilde{n}}\right]-\rho_{e} u_{e} \frac{d u_{e}}{d s}=\frac{\partial}{\partial \tilde{n}}\left(\mu_{T} \frac{\partial u}{\partial \tilde{n}}\right),
$$

where $u$ is aligned with the local surface tangent and $u>0$ when the flow is in the direction of increasing $s$. Here we assume that the flow in the viscous layer is adiabatic at unit Prandtl number; thus, the energy equation reduces to the requirement that the total enthalpy of the fluid, $H=T+u^{2} / 2$, must be constant across the viscous layer. In Eq. (4.3) the subscript $e$ refers to fluid properties at the edge of the viscous layer, and the effective turbulent viscosity, $\mu_{T}$, is defined by

$$
\mu_{T}=\mu+\gamma_{T} e,
$$

where $e$ is the turbulent eddy viscosity, $\gamma_{T}$ is the longitudinal intermittency factor and $\mu$ is the molecular viscosity, which is assumed to be a function of temperature alone. The eddy-viscosity model employed in the present study for blade surface boundary layers is that of Cebeci and Smith [20], modified to account for separated flow [7]; in the wake, the model of Chang, et al. [21] is used. In the present study, the location at which instantaneous transition occurs is specified.

The foregoing field equations govern the flow in the viscous layers along the upper and lower surfaces of the blades and in the blade wakes. They are solved subject to conditions at the edges of the viscous layer, on the airfoil surface, and along the reference wake streamline, i.e.,

$$
\begin{array}{cl}
u \rightarrow u_{e} & \text { for } \tilde{n} \rightarrow \infty, s \geq 0, \\
u=\tilde{v}=0 & \text { for } \tilde{n}=0,0 \leq s \leq s_{T E}^{ \pm}
\end{array}
$$

and

$$
\tilde{v}=0 \text { for } \tilde{n}=0, s>s_{T E}^{ \pm},
$$

respectively, where $s_{T E}^{ \pm}$are the trailing-edge values of the upper- $(+)$and lower-surface (-) arc-length coordinates measured from the leading-edge stagnation point. The condition expressed by Eq. (4.5) is also applied along the wake streamline for $\tilde{n} \rightarrow-\infty$. Equations (4.6) and (4.7) imply that the curve $\tilde{n}=0$ corresponds to the blade surfaces and reference wake streamlines, respectively.

The displacement and momentum thicknesses of the viscous layers ( $\delta$ and $\theta$, respectively) are needed to determine the effect of viscous displacement and wake curvature on the outer inviscid flow (Eqs. (3.3)-(3.5)). They are defined by

$$
\delta(s)=R e^{-1 / 2} \int_{0}^{\infty}\left(1-\frac{\rho u}{\rho_{e} u_{e}}\right) d \tilde{n}
$$


and

$$
\theta(s)=R e^{-1 / 2} \int_{0}^{\infty} \frac{\rho u}{\rho_{e} u_{e}}\left(1-\frac{u}{u_{e}}\right) d \tilde{n},
$$

where the zero lower bound on the integrals is replaced with $-\infty$ in the wake.

The independent and dependent variables appearing in the viscous-layer equations are transformed using a modified form [7] of the Levy-Lees transformation [22]. Thus, the independent variables are given by

$$
\xi=\int_{0}^{s} \rho_{e} u_{e} \mu_{e} e d s \text { and } \eta=\rho_{e} u_{e}(2 \xi)^{-1 / 2} \int_{0}^{\tilde{n}} \rho / \rho_{e} d \tilde{n},
$$

and the dependent variables are defined by

$$
F=u / u_{e} \text { and } f=(2 \xi)^{-1 / 2} \psi \text {. }
$$

The quantity $e$ is the value of the "outer" eddy-viscosity parameter (Eq. (4.4)), at each $s$-station, as defined in the Cebeci-Smith model [20]. It appears in the definition of $\xi$ in order to maintain a nearly constant $\eta$ value for the edge of the boundary layer in a turbulent flow [23]. The Levy-Lees transformation permits the leading-edge stagnation point similarity solution to be recovered and reduces the truncation error of the viscous solution over that associated with the use of primitive variables. The quantity $\psi$ is the compressible stream function defined in terms of the surface and wake streamline tangential and scaled normal velocity components $u$ and $\tilde{v}$, respectively, from the relations $\rho u=\partial \psi / \partial \tilde{n}$ and $\rho \tilde{v}=-\partial \psi / \partial s$.

The continuity and momentum equations transform to:

$$
F=\frac{\partial f}{\partial \eta}
$$

and

$$
\frac{\partial}{\partial \eta}\left(\ell \bar{\varepsilon} \frac{\partial F}{\partial \eta}\right)-2 \xi F \frac{\partial F}{\partial \xi}+\left(f+2 \xi \frac{\partial f}{\partial \xi}\right) \frac{\partial F}{\partial \eta}+\left(\hat{\theta}-F^{2}\right) \beta=0 .
$$

Here $\ell=\rho \mu / \rho_{e} \mu_{e}, \bar{\varepsilon}$ is the ratio of the turbulent to molecular viscosity coefficients and $\beta$ is the pressure gradient parameter, defined by

$$
\beta=\frac{2 \xi}{u_{e}} \frac{d u_{e}}{d \xi} .
$$

The quantity $\hat{\theta}$ is equal to $T / T_{e}$, the local to edge static temperature ratio, which is related to $F$ as follows:

$$
\hat{\theta}=1+\frac{\gamma-1}{2} M_{e}^{2}\left(1-F^{2}\right) .
$$

The molecular viscosity coefficient, $\mu$, is determined using the Sutherland viscosity law; i.e.,

$$
\frac{\mu}{\mu_{-\infty}}=\left(\frac{T}{T_{-\infty}}\right)^{3 / 2} \frac{T_{-\infty}+T_{C}}{T+T_{C}} .
$$

Here $\mu_{-\infty}$ is the molecular viscosity at the temperature $T_{-\infty}$ and $T_{C}$ is a constant, which for air has a dimensional value of $110^{\circ} \mathrm{K}$ [24]. 
The following boundary conditions are applied: the blade and wake reference surfaces (at $\eta=0$ ) are both streamlines, therefore the stream function is a constant, so that, without loss of generality, we can set

$$
f=0 \quad \text { at } \quad \eta=0 .
$$

On the blade surface the no-slip condition applies so that

$$
F=0 \quad \text { at } \quad \eta=0, \xi \leq \xi_{T E},
$$

where $\xi_{T E}$ is the trailing-edge value of $\xi$. At the edges of the surface and wake viscous layers the remaining boundary conditions are applied, namely

$$
F\left(\eta_{e}^{+}\right)=F\left(\eta_{e}^{-}\right) \equiv 1,
$$

where $\eta_{e}^{+}$and $\eta_{e}^{-}$are the upper and lower viscous-layer edge values of the wake $\eta$-coordinate, respectively; the \pm superscripts are not needed for the surface boundary layers since each has only one edge. The boundary condition given by Eq. (4.19) enforces the approach of the flow variables to their correct edge values. Equation (4.19) is consistent with the assumption that the static pressure jump across the wake is negligible.

The viscous-layer equations are parabolic in the $\xi$-direction and therefore require initial conditions. These are provided by a similarity solution obtained at the leading-edge stagnation point, $\xi=0$, by solving the above equations with $\beta=1$. The blade surface and wake solutions are obtained by space-marching in the downstream direction. As discussed in $\S 2$, a complete IVI calculation requires the ability to solve the viscous-layer equations in both the "direct" mode, where the pressure gradient parameter $\beta$ is specified and the displacement thickness $\delta$ is unknown, and in the "inverse" mode, where $\delta$ is specified and $\beta$ is unknown. In particular, an inverse calculation is required to predict viscous-layer separation. The equations are solved in the direct mode near the leading edge, and in the inverse mode downstream of an axial station chosen to ensure that the inverse mode is initiated upstream of a separation point. The wake is calculated using the inverse mode.

In the direct viscous-layer calculation, the value of $\beta$ is determined by the inviscid analysis and the displacement thickness is obtained from the viscous analysis. In the inverse procedure, the value of the mass deficit parameter, $\bar{m}=\rho_{e} u_{e} \delta$, is specified and the edge values of the variables $u_{e}, M_{e}$, etc. are obtained as part of the viscous-layer solution. An expression for $f$ at the edge of the viscous layer is derived by integrating Eq. (4.12) across the boundary layer and employing the definition of $\delta$ (Eq. (4.8)), i.e.,

$$
f\left(\eta_{e}\right)=h+\eta_{e}-\frac{\bar{m}}{\sqrt{2 \xi}},
$$

where

$$
h=\int_{0}^{\eta_{e}}(\hat{\theta}-1) d \eta .
$$

Equation (4.20) is used on blade surfaces to impose the specified value of $\bar{m}$ through the corresponding value of $f$ at the outer edge of the viscous mesh, $\eta=\eta_{e}$. The parameter $h$ is specified, and is lagged from the previous global iteration. Similarly, in the wake an expression for the jump in the stream function between the viscous-layer upper and lower 
edges is obtained by integrating Eq. (4.12) across the entire wake (from $\eta_{e}^{-}$to $\eta_{e}^{+}$) and using the definition of the displacement thickness, i.e.,

$$
f\left(\eta_{e}^{+}\right)-f\left(\eta_{e}^{-}\right)=h_{\mathcal{W}}+\eta_{e}^{+}-\eta_{e}^{-}-\frac{\bar{m}_{\mathcal{W}}}{\sqrt{2 \xi}},
$$

where

$$
h_{\mathcal{W}}=\int_{\eta_{e}^{-}}^{\eta_{e}^{+}}(\hat{\theta}-1) d \eta .
$$

Equations (4.22) and (4.23) are used in the same way that the corresponding equations were used on the surfaces to impose $\bar{m}_{\mathcal{W}}=\left(\rho_{e} u_{e} \delta\right)_{\mathcal{w}}$ and $h_{\mathcal{W}}$. In the inverse mode the quantities $\beta$ and $u_{e}$ are unknown. Thus, a supplemental equation relating these two variables is needed. This relation is obtained by discretizing Eq. (4.14), which defines $\beta$ in terms of $u_{e}$.

The discretized governing equations, boundary and auxiliary conditions, Eqs. (4.12)(4.23), are quasi-linearized and the resulting coupled tridiagonal system of algebraic equations is solved at each $s$-station, using a fixed-point iteration to update the nonlinear terms. The inversion algorithm used in the wake is modified to account for the application of one boundary condition (Eq. (4.17)) at $\eta=0$ and the others (Eq. (4.19)) at the upper and lower edges of the viscous layer, as well as to account for the application of a jump condition on $f$ (Eq. (4.22)) between the upper and lower edges. Finally, the so-called FLARE approximation, which prevents instabilities in the viscous-layer solution due to axial flow reversal, is applied by turning off all of the convective terms in the momentum equation wherever $F<0$. The details of the viscous-layer numerical analysis are provided in Appendix A of this report. 


\section{Inviscid/Viscid Interaction}

The IVI approach used here determines the complete flow field by iteratively updating the mass deficit parameter, $\bar{m}(s)$, which affects the inviscid and viscous solutions through their respective boundary conditions. For an arbitrary $\bar{m}$ distribution, two different surface and wake streamline velocity distributions generally result - one, $u_{e_{I}}(s)$, from the inviscid calculation and one, $u_{e_{V}}(s)$, from the viscous-layer calculation. The objective is to determine a converged inviscid/viscid interaction solution by finding the mass deficit parameter distribution that minimizes the difference between the $u_{e_{I}}$ and $u_{e_{V}}$ distributions. In this investigation the so-called "semi-inverse" iteration procedure of Carter [25] is used to update $\bar{m}$ at every streamwise mesh station on the blade and wake surfaces. The term "semi-inverse" refers to the use of an inverse method (i.e., displacement thickness specified and pressure determined) to solve the viscous-layer equations coupled to a direct method (i.e., geometry specified and pressure determined) to solve the inviscid equations. We set

$$
\bar{m}^{n+1}=\bar{m}^{n}\left[1+\omega\left(u_{e_{V}}^{n} / u_{e_{I}}^{n}-1\right)\right],
$$

where the superscript $n$ is the global iteration count and $\omega$ is a relaxation parameter. The solution is considered to be converged when

$$
\max _{i}\left|u_{e V_{i}}-u_{e_{I_{i}}}\right| / u_{e_{I_{i}}}<\hat{\epsilon}, i=1, \ldots, I E,
$$

where the value of $\hat{\epsilon}$ is specified by the user and $I E$ is the number of streamwise mesh stations. Equation (5.2) is applied on both blade surfaces and along the wake. The viscouslayer solution is obtained at the locations corresponding to the intersections of the inviscid mesh with the blade and wake streamline surfaces, which avoids the need for interpolation.

Because a major objective of this study is to develop an efficient analysis, various techniques for accelerating convergence were examined. We found that one of the most effective approaches for reducing the CPU time needed to obtain a converged IVI solution is to use the largest value of the relaxation parameter $\omega$ for which the iterative procedure remains stable. It was observed during this study that the inviscid surface velocity distribution, $u_{e_{I}}$, undergoes relatively little change from the initial purely inviscid solution to the final converged IVI solution. This is in contrast to the significantly larger change between the initial and final viscous-layer edge distribution, $u_{e_{V}}$. This observation prompted the introduction of a subiteration loop in which the viscous equations are solved repeatedly (" $N_{v}$ " times) during each global iteration. Thus, Eq. (5.1) is applied $N_{v}$ times during a single global iteration with $u_{e_{I}}$ frozen at its most recent value while $u_{e_{V}}$ is recalculated during each subiteration by solving the viscous-layer equations using the latest $\bar{m}$ distribution. The value of $N_{v}$ is a user-specified input; the standard iteration procedure is recovered when $N_{v}=1$. This strategy is only effective in reducing the total CPU time if the number of global iterations needed to obtain a converged IVI solution, $N_{g}$, can be reduced enough to more than balance the increased computational effort needed for the additional viscous calculations performed during each global iteration. Of the three cascade configurations examined in $\S 6$, only the turbine cascade benefitted from this approach, as discussed in the next section.

The semi-inverse iteration procedure is illustrated in Fig. 2, where the dashed line corresponds to the viscous subiteration technique described above. 


\section{Numerical Examples}

The foregoing inviscid/viscid interaction analysis has been applied to the following cascade configurations: a compressor exit guide vane (EGV), a high-speed compressor (HSC) and a turbine cascade. For the compressor cascades the behavior of the surface and wake pressure coefficient, $C_{P}=\left(P-P_{-\infty}\right) / 2$ will be examined. The surface Mach number distribution will be examined for the turbine cascade. The displacement thickness, $\delta$, and the surface shear stress, $\tau_{w}=R e^{-1} \mu \partial u /\left.\partial n\right|_{n=0}$, distributions will be presented for all three configurations. The IVI solutions for the compressor cascades will be evaluated through comparisons with Navier-Stokes solutions; that for the turbine with experimental measurements. In addition, the predicted values of the total pressure loss and exit flow angle are presented for the EGV cascade. These were obtained using the mixing analysis of Stewart [28]. Alternatively, total pressure loss and exit flow angle could be based on the predicted average flow conditions at the exit plane of the computational domain. Both methods have been implemented in SFLOW-IVI and give results that are in very good agreement for the flows considered in this study. Finally, the performance of the SFLOW-IVI analysis, i.e., its efficiency and convergence properties, will also be discussed.

In all of the calculations described here, the SFLOW-IVI analysis was applied using a convergence tolerance, $\hat{\epsilon}$, of 0.001 (see Eq. (5.2)). The inviscid meshes that were used for both compressor cascades have the same dimensions, i.e., there are 90 axial and 31 circumferential lines, with 24 axial lines upstream of the leading edge, 41 lines intersecting the blade surfaces and 25 lines aft of the trailing edge. The viscous-layer analysis employed a total of 81 blade and 25 wake streamwise grid lines, with 71 grid lines across the surface boundary layer and 141 grid lines across the wake. The inviscid mesh used for the turbine cascade had 150 axial and 31 circumferential lines, with 39 points upstream of the leading edge, 51 points along each blade surface and 60 points along the wake. A total of 101 surface and 25 wake stations were used in the viscous-layer analysis. The normal mesh had the same dimensions as those used for the compressor cascades. For the cases considered in this study, the wake curvature effect was regarded to be negligible; thus, $\llbracket P \rrbracket_{w}$ was set equal to zero, cf Eq. (3.5).

The inviscid solutions were obtained on a "streamline" type H-mesh, rather than on the "sheared" H-mesh described in Refs. [14] and [17]. The SFLOW analysis of Ref. [17] was modified to use the streamline H-mesh developed by Hall and Verdon [26]. Thus, prior to initiating an IVI calculation, an inviscid solution is obtained on a sheared $\mathrm{H}$-mesh. This solution is then used to generate a streamline $\mathrm{H}$-mesh, in which one family of mesh lines corresponds to the streamlines of the inviscid flow solution obtained using the simple sheared H-type mesh, and the second family consists of lines which are "nearly" orthogonal to the first set. The principal advantage of the streamline H-mesh over the sheared mesh is an improved resolution of the flow in the blade leading-edge regions.

An alternative to this procedure is available in SFLOW and was used for one of the cases described below, i.e., the turbine cascade. In the turbine case, a useful streamline $\mathrm{H}$-mesh could not be produced from the solution obtained using a sheared $\mathrm{H}$-mesh, because the latter provides inadequate resolution of the leading-edge region. To remedy this, the initial inviscid solution was obtained on a "composite" mesh constructed by overlaying a surface fitted C-mesh, generated locally in the leading-edge region, over the global sheared 
H-mesh. A detailed description of this procedure, as applied to linearized unsteady flows, can be found in Ref. [27].

\subsection{Compressor Exit Guide Vane}

The EGV cascade consists of 12 percent thick, highly cambered, modified NACA airfoils [26]. It has a stagger angle, $\Theta$, of $15 \mathrm{deg}$, a gap-chord ratio, $G$, of 0.6 and operates at a prescribed inlet Mach number, $M_{-\infty}$, and inlet flow angle, $\Omega_{-\infty}$, of 0.3 and 40 deg, respectively. Calculations were performed for an inviscid flow, and for viscous flows at Reynolds numbers of $10^{5}$ and $10^{6}$. Instantaneous transition from laminar to turbulent flow was assumed to occur at one percent of the arc distance measured from the leading-edge stagnation point to the trailing edge on both the suction and pressure surfaces of the blades. This is a physically realistic assumption for most high Reynolds number flows and is consistent with the usual practice in high $R e$ Navier-Stokes calculations in which instantaneous transition is assumed to occur at blade leading edges. A streamline mesh is depicted in Fig. 3, where three adjacent EGV blade passages are shown. For the purpose of illustration, the mesh shown in this figure has approximately half as many axial and circumferential grid lines than were used for the actual calculations.

Results of the inviscid and IVI calculations are shown in Fig. 4. The blade and wake pressure and displacement thickness distributions are shown in Figs. 4(a) and 4(b), respectively, and the surface shear-stress distributions along the blade in Fig. 4(c). The expected approach of the viscous to the inviscid solution as Re is increased is evident in Fig. 4(a). The rate of growth of the suction-surface displacement thickness increases with increasing $x$ as the viscous-layer separation point is approached. In the wake the half-wake displacement thickness, $\delta_{\mathcal{W}} / 2$ is plotted in Fig. $4(\mathrm{~b})$. As expected, its value just aft of the trailing edge is approximately equal to the mean of the upper- and lower-surface trailing-edge displacement thicknesses. As shown in Fig. 4(c), a suction-surface separation bubble $\left(\tau_{w}<0\right)$ exists and spans approximately 14 percent of chord at $R e=10^{6}$, and about 24 percent of chord for $R e=10^{5}$. The decrease in the extent of the separation bubble as $R e$ is increased is consistent with the behavior expected for turbulent flows.

The surface pressure, displacement thickness and surface shear-stress distributions predicted by SFLOW-IVI are compared in Fig. 5 with results obtained using the Navier-Stokes analysis of Dorney, et al. [29] for the $R e=10^{6}$ case. The N-S analysis uses the BaldwinLomax turbulence model [30], which is very similar to the Cebeci-Smith model used in SFLOW-IVI. Good agreement between the results of the two procedures is obtained over most of the blade surface for all three quantities. However, the agreement deteriorates in the vicinity of the trailing edge. This is caused by the use of an $\mathrm{O}$-mesh around the blades in the Navier-Stokes analysis. The O-mesh topology is not well-suited to wedge-shaped trailing edges like those found in the EGV cascade. Both analyses predict separation $\left(\tau_{w}<0\right)$ near the trailing edge, and give almost identical predictions for the location of the separation point $\left(\tau_{w}=0\right.$; see Fig. $\left.5(\mathrm{c})\right)$.

To test the robustness of the SFLOW-IVI analysis, additional calculations were carried out for $M_{-\infty}=0.3, R e=10^{6}$ and a wide range of inlet flow angles, $36 \mathrm{deg} \leq \Omega_{-\infty} \leq 54 \mathrm{deg}$. The transition point locations were held fixed at $s / s_{T E}=0.01$ for all values of $\Omega_{-\infty}$. This location is the same as that reported above for the baseline $\left(\Omega_{-\infty}=40 \mathrm{deg}\right)$ calculation. 
The results are shown in Fig. 6, where the predicted total pressure loss parameter, $\bar{\omega}=$ $\left(P_{t_{-\infty}}-P_{t_{+\infty}}\right) /\left(P_{t_{-\infty}}-P_{-\infty}\right)$ (where $P_{t}$ is the total pressure), exit flow angle, $\Omega_{+\infty}$, and separation point location, $x_{\text {sep }}$, are plotted as functions of $\Omega_{-\infty}$. Solutions could not be obtained for $\Omega_{-\infty}<36$ deg because a small supersonic region, which could not be treated using the present version of SFLOW-IVI, formed near the leading edge of each blade. At $\Omega_{-\infty}=54 \mathrm{deg}$, the viscous layer is approaching stall, with the separation region spanning approximately 35 percent of chord. Above $54 \mathrm{deg}$ the solution would not converge due to a numerical instability. This is consistent with the known stability properties of the semiinverse IVI iteration procedure when applied to flows with large-scale separations [31].

The total pressure loss parameter and the exit flow angle are plotted versus $\Omega_{-\infty}$ in Figs. 6(a) and 6(b), respectively. There is a wide range of inlet flow angles over which the loss remains relatively low, while $\bar{\omega}$ increases rapidly as the inlet flow angle is increased above $50 \mathrm{deg}$. The latter corresponds to the rapid inflation of the separation region with increasing $\Omega_{-\infty}$ for $\Omega_{-\infty}>50 \mathrm{deg}$, which can be seen in Fig. 6(c). A striking similarity exists between the variations in $-\Omega_{+\infty}$ and $x_{\text {sep }}$ as $\Omega_{-\infty}$ is varied, as is apparent from comparing the results shown in Figs. 6(b) and 6(c). The streamwise growth of the separation bubble as $\Omega_{-\infty}$ is increased is accompanied by a similar increase in the suction-surface displacement thickness in the vicinity of the trailing edge. This produces a thickened displacement body (i.e., blade plus displacement thickness), reducing the effective camber of the blade and thus, the loading it produces. As a direct consequence there is a reduction in the turning of the flow, i.e., an increase in $\Omega_{+\infty}$.

The predicted streamline patterns indicating the size of the trailing-edge separation bubble for $\Omega_{-\infty}=36 \mathrm{deg}, 45 \mathrm{deg}$ and $54 \mathrm{deg}$ are shown in Fig. 7. The separation bubble grows slowly in the range $36 \leq \Omega_{-\infty} \leq 45 \mathrm{deg}$, and much more rapidly between 45 and 54 deg. The "decambering" effect produced by the growth of the separation bubble is clearly illustrated by these results. The kinks that appear in the streamlines near the trailing edge, depicted in Fig. 7, require some explanation. Since the blade trailing edge is wedge-shaped, the surface coordinate line formed by the blade surface and reference wake streamline has a geometric singularity or "kink" at the trailing edge. This singularity influences the solution throughout the trailing-edge region as shown in the streamline plots in Fig. 7. Because this singular behavior is highly localized, its effect on the overall flow field solution is negligible. An additional factor contributing to the kinks is the use of a relatively coarse streamwise mesh aft of the trailing edge. This results in an abrupt change in the flow variables across the trailing-edge point.

\subsection{High-Speed Compressor}

The HSC cascade consists of cambered, modified NACA 0006 airfoils [32]. This cascade operates at high-subsonic inlet conditions, i.e., $M_{-\infty}=0.7$ and $\Omega_{-\infty}=55 \mathrm{deg}$, and has a blade spacing and a stagger angle of unity and $45 \mathrm{deg}$, respectively. As was done for the EGV cascade, two values of the Reynolds number have been considered, $10^{5}$ and $10^{6}$. Instantaneous transition was assumed to occur at ten and at one percent of the surface arc length for $R e=10^{5}$ and $10^{6}$, respectively, on both the suction and pressure surfaces of each blade. The HSC cascade solution for $R e=10^{5}$ was found to be sensitive to the specified location of transition. The solution could not be converged with transition specified at 
one percent of arc length, however, specifying transition farther downstream permitted the solution to converge. The reason for this sensitivity has not yet been determined and needs to be examined in future work. A mesh which has, for the sake of clarity, a lower grid point density than was used for the actual calculations is shown in Fig. 8. Inviscid and viscous calculations were performed and the results are presented in Figs. 9 and 10.

The predicted pressure and displacement thickness distributions along the blade surfaces and the wake are shown in Figs. 9(a) and 9(b), respectively. The behavior of both quantities is similar to that observed for the EGV. The surface shear-stress distributions shown in Fig. 9(c) indicate that the extents of the suction-surface separation bubbles are smaller than those predicted for the EGV cascade, decreasing from approximately 20 percent to about 8 percent of chord as the Reynolds number is increased from $10^{5}$ to $10^{6}$. The kinks in the shear-stress distributions for the $R e=10^{5}$ case are associated with transition.

The surface pressure coefficient, displacement thickness and shear-stress distributions obtained for $R e=10^{6}$ using SFLOW-IVI are compared in Fig. 10 with those obtained using the Navier-Stokes analysis of Ref. [29]. The agreement is excellent except in the immediate vicinity of the trailing edge. The two analyses give almost identical predictions for the location of the separation point. Again, the differences in the two solutions are attributed to the use of an $\mathrm{O}$-mesh for wedge-shaped trailing-edge geometries in the Navier-Stokes analysis.

\subsection{Turbine}

The turbine cascade considered here is the Fourth Standard Configuration described in the study of Fransson and Suter [33]. The blade geometry is shown in Fig. 11 and was obtained by modifying the original blunt trailing-edge geometry to produce a wedge-shaped trailing edge while retaining the original chord length, as discussed in Ref. [17]. As for the cases discussed above, the mesh shown in the figure has fewer grid lines than were used in the actual calculation. The streamline mesh employed for the turbine calculation was obtained from an inviscid solution calculated using the composite mesh analysis discussed in $\S 4$ and in Ref [27].

The blade spacing and stagger angle for the turbine cascade are 0.76 and $56.6 \mathrm{deg}$, respectively, and the inlet Mach number and flow angle are 0.205 and $45 \mathrm{deg}$, respectively. The value of $M_{-\infty}$ has been adjusted from the experimentally measured value of 0.190 to improve the agreement with the measured pressure distribution. The calculation was carried out at a Reynolds number of $5 \times 10^{5}$ with instantaneous transition occurring at 10 percent of the surface arc length along both surfaces. A converged solution could not be obtained for the turbine cascade if the location of transition was specified to be too close to the leading edge. This was consistent with the behavior observed for the HSC cascade calculation at $R e=10^{5}$.

The IVI solution was obtained in 12 global inviscid/viscid iterations. The viscous subiteration procedure described in $\S 5$ was very effective for this case, reducing the CPU time needed to converge the calculation from 1371 seconds without subiteration (requiring 115 global iterations) to 224 seconds (in 12 global iterations), using four viscous subiterations (i.e., $\left.N_{v}=4\right)$ during each global IVI iteration.

The computed and measured blade surface Mach number distributions are shown in 
Table 1. Summary of SFLOW-IVI CPU times, $t_{C}$, for different cascade configurations.

\begin{tabular}{|c|c|c|c|c|}
\hline Configuration & $\omega$ & $N_{v}$ & $N_{g}$ & $t_{C}($ secs $)$ \\
\hline \hline EGV,$R e=10^{6}$ & 1.20 & 1 & 24 & 197 \\
\hline EGV, $R e=10^{5}$ & 0.85 & 1 & 38 & 277 \\
\hline HSC, $R e=10^{6}$ & 1.20 & 1 & 27 & 203 \\
\hline HSC, $R e=10^{5}$ & 0.80 & 1 & 40 & 296 \\
\hline Turbine & 0.55 & 4 & 12 & 224 \\
\hline
\end{tabular}

Fig. 12(a). Viscous effects produce a nearly uniform decrease in the suction-surface Mach number distribution aft of $x \approx 0.4$, while the pressure-surface distribution is almost unaffected. The agreement between the IVI solution and the experimental data is reasonable the disagreement in the trailing-edge region may be partly attributable to the local geometry modification mentioned above. It is difficult to draw definitive conclusions regarding the experimental comparison because the solution for this case is particularly sensitive to the inviscid mesh. The predicted displacement thickess and surface skin-friction coefficient distributions are shown in Figs. 12(b) and 12(c), respectively. No separation was predicted for the conditions considered here, although the suction-surface viscous-layer is close to separation at the trailing edge.

\subsection{Timing Study and Convergence Behavior}

Because the development of an efficient analysis has been a major objective of this analytical effort, a timing study was conducted for the three cascade configurations examined here. This provides both a measure of the computational effort presently required to obtain solutions using SFLOW-IVI, and benchmarks against which future efforts to improve efficiency can be compared. The results are summarized in Table 1 . In addition to the CPU time $\left(t_{C}\right)$, the relaxation factor $(\omega)$, the number of viscous subiterations used $\left(N_{v}\right)$ and the number of global iterations $\left(N_{g}\right)$ required to converge the IVI solution using a tolerance level, $\hat{\epsilon}$, of 0.001 , are given in Table 1 . The execution times were determined using the nearly optimal value of $\omega$, which was obtained by trial and error.

The calculations were carried out on an HP-Apollo 720 workstation where SFLOW-IVI has been compiled using an optimizing preprocessor. No attempt has been made to "tune" the code to take advantage of special features of the optimizer. The times given in Table 1 are CPU times for the portion of the calculation associated with the IVI iteration loop. Any overhead associated with initialization of the data structure, generation of the mesh and calculation of the initial inviscid solution is not included. However, this overhead amounts to a small percentage of the overall CPU time required by the present analysis. Note that each of the solutions was obtained in less than five minutes.

The convergence behavior of two parameters of interest to compressor blade designers has been examined here to determine if the solutions discussed above are sufficiently con- 
verged, and whether a different measure of convergence than that given by Eq. (5.2) would be more appropriate. For the two compressor cascades, the total pressure loss parameter $\bar{\omega}$ and exit flow angle $\Omega_{+\infty}$ were monitored during the IVI iterations. We have found that, for most engineering purposes, the solutions obtained herein could be considered converged at a significantly lower iteration count than was needed to satisfy the convergence criterion $(\hat{\epsilon}=0.001)$. Thus, even greater efficiency could be achieved in many cases by measuring convergence by the degree to which the parameters of interest have approached their "asymptotic" values. This is demonstrated by the results presented in Fig. 13, which show the behavior of $\bar{\omega}$ and $\Omega_{+\infty}$, respectively, as functions of the iteration count for the EGV cascade operating at $R e=10^{6}$ and $\Omega_{-\infty}=40 \mathrm{deg}$. This behavior is typical of that observed for all of the cases studied herein. The solution for the case illustrated in Fig. 13 converged to $\hat{\epsilon}=0.001$ within 24 iterations while the asymptotic value, indicated by the dashed horizontal line, was determined by converging the solution to $\hat{\epsilon}=0.0001$, for which 41 iterations were needed. For engineering purposes, this solution could be considered to be converged after about 15 iterations, for which $t_{C}$ is approximately 120 seconds.

The CPU times required to obtain the present IVI solutions are significantly lower than those needed to obtain Navier-Stokes solutions of comparable accuracy. The latter currently require approximately one to three hours of CPU time on a modern workstation. However, to achieve such CPU times considerably fewer grid points (typically between 8 and 15) are used across the surface boundary layers than are used in IVI calculations. For example, 71 points were used for the SFLOW-IVI calculations presented in this report. Thus SFLOW-IVI requires one to two orders-of-magnitude less CPU time than current Navier-Stokes analyses to achieve viscous solutions for 2-D cascade flows. 


\section{Concluding Remarks}

In the present study, existing nonlinear inviscid and inverse viscous-layer analyses have been extended and coupled to provide a strong inviscid/viscid interaction solution capability for two-dimensional cascade flows. This IVI solution procedure can be used to predict the effects of local strong interactions, including trailing-edge/near-wake interactions and smallto moderate-scale viscous-layer separations, on cascade performance. The present analysis is restricted to subsonic flows, but it can be extended to treat transonic flows.

The SFLOW-IVI analysis has proven to be accurate, efficient and robust. During this study the analysis was applied to compressor exit guide vane, high-speed compressor and turbine cascades. The analysis has been shown to be able to predict both the detailed features of the flow field as well as global quantities such as loss and turning. Very good agreement with Navier-Stokes solutions was demonstrated for two of the cases considered herein.

Converged solutions for each of the foregoing configurations examined here were obtained in less than five minutes on an HP-Apollo 720 Workstation. It was shown that even lower CPU times could be obtained by basing convergence on the global quantities of interest to the engine designer. The robustness of the SFLOW-IVI analysis was demonstrated through application to a wide range of inlet conditions, including cases in which large-scale separations, spanning up to 35 percent of chord, occurred. It should be noted that CPU times for the most severe cases were on the order of 15 to 20 minutes.

A number of issues still need to be addressed in order to improve the accuracy of the present steady IVI analysis, and to expand its range of applicability. Among them are the inclusion of quasi-three-dimensional effects (i.e., streamtube contraction and radius change), the incorporation of predictive models for determining the transition from laminar to turbulent flow and the addition of a procedure for updating the location of the wake streamline during the global iteration process. With respect to transition, at present we are specifying the transition location, but for a truly predictive viscous calculation (either IVI or NavierStokes) the transition location should be determined as part of the complete solution. In addition, the overall utility of the SFLOW-IVI analysis for design-system applications needs to be explored through further testing and validation. Finally, as this effort continues, the focus will increasingly turn towards the development of an unsteady strong inviscid/viscid interaction capability based, as much as possible, on the complementary SFLOW-IVI and LINFLO analyses. 


\section{References}

1. Melnik, R. E., "Turbulent Interactions on Airfoils at Transonic Speeds - Recent Developments," AGARD-CP-291, Chapter 10, 1980.

2. Lock, R. C. and Firmin, M. C. P., "Survey of Techniques for Estimating Viscous Effects in External Aerodynamics," Royal Aircraft Establishment Technical Memorandum Aero 1900, April 1981.

3. Girodroux-Lavigne, P. and Le Balleur, J. C., "Unsteady Viscous-Inviscid Interaction Method and Computation of Buffeting over Airfoils," ONERA T.P. No. 1987-58, 1987.

4. Howlett, J. T., "Efficient Self-Consistent Viscous-Inviscid Solutions for Unsteady Transonic Flow, AIAA Paper 85-0482, 1985.

5. Caspar, J. R., "Unconditionally Stable Calculation of Transonic Potential Flow through Cascades Using an Adaptive Mesh for Shock Capture," ASME Journal of Engineering for Power, Vol. 105, No. 3, 1983, pp. 504-513.

6. Vatsa, V. N. and Verdon, J. M., "Viscous/Inviscid Interaction Analysis of Separated Trailing-Edge Flows," AIAA Journal, Vol. 23, No. 4, 1985, pp. 481-489.

7. Barnett, M. and Verdon, J. M., "Viscid/Inviscid Interaction Analysis of Subsonic Turbulent Trailing-Edge Flows," AIAA Journal, Vol. 25, No. 9, September 1987, pp. 1184-1193.

8. Veldman, A. E. P., "The Calculation of Incompressible Boundary Layers with Strong Viscous-Inviscid Interaction," AGARD-CP-291, Chap. 12, 1980.

9. Hansen, E. C., Serovy, G. K. and Sockol, P. M., "Axial-Flow Compressor Turning Angle and Loss by Inviscid-Viscous Interaction Blade-to-Blade Computation," ASME Paper No. 79-GT-5, 1979.

10. Janssens, P. and Hirsch, Ch., "A Viscid Inviscid Interaction Procedure for Two-Dimensional Cascades," AGARD-CP-351, Chapter 3, 1983.

11. Calvert, W. J. and Herbert, M. V., "An Inviscid-Viscous Interaction Method to Predict the Blade-to-Blade Performance of Axial Compressors," Aeronautical Quarterly, Vol. XXXI, Part 3, 1980, pp. 173-196.

12. Barnett, M. and Verdon, J. M., "Theoretical Prediction of High Reynolds Number Viscid/Inviscid Interaction Phenomena in Cascades," Proceedings of the Fourth Symposium on Numerical and Physical Aspects of Aerodynamic Flows, Long Beach, California, 1989.

13. Barnett, M., Hobbs, D. E. and Edwards, D. E., "Inviscid-Viscous Interaction Analysis of Compressor Cascade Performance," ASME Journal of Turbomachinery, Vol. 113, No. 4, 1991, pp. 538-553.

14. Verdon, J. M. and Caspar, J. R., "A Linearized Unsteady Aerodynamic Analysis for Transonic Cascades," Journal of Fluid Mechanics, Vol. 149, 1984, pp. 403-429. 
15. Power, G. D., Verdon, J. M. and Kousen, K. A., "Analysis of Unsteady Compressible Viscous Layers," ASME Journal of Turbomachinery, Vol. 113, 1991, pp. 644-653.

16. Barnett, M. and Verdon, J. M., "Analysis of Blade Unsteady Boundary Layers and Wakes," presented at the Sixth International Symposium on Unsteady Aerodynamics, Aeroacoustics and Aeroelasticity of Turbomachines and Propellers, Notre Dame, Indiana, September 1991; Proceedings to be published by Springer-Verlag.

17. Hoyniak, D. and Verdon, J. M., "Development of a Steady Potential Solver for Use With Linearized, Unsteady Aerodynamic Analyses," NASA TM 105288, September 1991.

18. Goldstein, S. "On Laminar Boundary Layer Flow Near a Position of Separation," Quarterly Journal of Mechanics and Applied Mathematics, Vol. 1, 1948, pp. 43-69.

19. Stewartson, K., "Is the Singularity at Separation Removable?" Journal of Fluid Mechanics, Vol. 44, Part 2, 1970, pp. 347-364.

20. Cebeci, T. and Smith, A. M. O., Analysis of Turbulent Boundary Layers, Academic Press, New York, 1974.

21. Chang, K. C., Bui, M. N., Cebeci, T. and Whitelaw, J. H., "The Calculation of Turbulent Wakes," AIAA Journal, Vol. 24, No. 2, 1986, pp. 200-201.

22. Blottner, F. G., "Finite Difference Method of Solution of the Boundary-Layer Equations," AIAA Journal, Vol. 18, No. 2, 1970, pp. 193-205.

23. Vatsa, V.N., Werle, M.J. and Verdon, J.M., "Viscid/Inviscid Interaction at Laminar and Turbulent Symmetric Trailing Edges," AIAA Paper 82-0165, 1982.

24. Schlichting, H., Boundary-Layer Theory, Seventh Edition, McGraw-Hill Book Company, Inc., New York, 1979, p. 328.

25. Carter, J. E., "A New Boundary Layer Inviscid Iteration Technique for Separated Flow," AIAA Paper 78-1450, 1978.

26. Hall, K. C. and Verdon, J. M., "Gust Response Analysis for Cascades Operating in Nonuniform Mean Flows," AIAA Journal, Vol. 29, No. 9, 1991, pp. 1463-1471.

27. Usab, W. J., Jr. and Verdon, J. M., "Advances in the Numerical Analysis of Linearized Unsteady Cascade Flows," ASME Journal of Turbomachinery, Vol. 113, 1991, pp. 633643.

28. Stewart, W.L., "Analysis of Two-Dimensional Compressible Flow Loss Characteristics Downstream of Turbomachine Blade Rows in Terms of Basic Boundary-Layer Characteristics," NACA TN 3515, 1955.

29. Dorney, D.J., Davis, R.L. and Edwards, D.E., "Investigation of Hot Streak Migration and Film Cooling Effects on Heat Transfer in Rotor/Stator Interacting Flows," UTRC Report 91-29, April 1992. Prepared under NAVAIR Contract N00140-88-C-0677 (Report 1). 
30. Baldwin, B. S. and Lomax, H., "Thin-Layer Approximation and Algebraic Model for Separated Turbulent Flows," AIAA Paper 78-257, 1978.

31. Wigton, L.B. and Holt, M., "Viscous-Inviscid Interaction in Transonic Flow," AIAA Paper 81-1003, 1981.

32. Verdon, J. M. and Hall, K. C., "Development of a Linearized Unsteady Aerodynamic Analysis for Cascade Gust Response Predictions," NASA CR 4308, July 1990.

33. Fransson, T. H. and Suter, P., "Two-Dimensional and Quasi Three-Dimensional Experimental Standard Configurations for Aeroelastic Investigations in Turbomachine Cascades," Report LTA-TM-83-2, Ecole Polytechnique Federale De Lausanne, Lausanne, Switzerland, 1983.

34. Reyhner, T. A. and Flügge-Lotz, I., "The Interaction of a Shock Wave with a Laminar Boundary Layer," Int. J. Non-Linear Mech., Vol. 8, No. 2, 1968, pp. 173-193. 


\section{List of Symbols}

All physical parameters listed below are dimensionless, as described in $§ 3$, General Concepts. The number(s) in parentheses at the end of each symbol description indicates an equation in which the symbol appears.

\section{$\underline{\text { Roman }}$}

A Speed of sound propagation, (3.1).

$C_{P} \quad$ Pressure coefficient, Figure 4.

e Turbulent eddy viscosity, (4.4).

$F, f$ Dependent variables in Levy-Lees transformation, (4.11).

G Cascade gap-to-chord ratio, Figure 1.

H Total enthalpy.

h Viscous-layer integral parameter, (4.21).

$\ell \quad$ Viscous-layer parameter, (4.13).

M Mach number, (3.2).

$\bar{m} \quad$ mass deficit parameter, (4.20), (4.22).

$N_{g} \quad$ Number of global iterations to converge solution, Table 1.

$N_{v} \quad$ Number of viscous subiterations per global iteration.

$\tilde{n}$ Scaled viscous-layer normal distance from surface and wake reference streamline, (4.1).

n Unit normal positive when directed outward from blade surface or upward from wake reference streamline, (3.3), (3.4).

$P \quad$ Pressure, (3.2).

Re Reynolds number.

$\mathcal{S} \quad$ Blade surface, Figure 1. 
$s \quad$ Surface and wake streamline arc-distance measured from leading-edge stagnation point, $(4.2,4.3)$.

T Temperature, (3.2).

$t_{C} \quad$ CPU time to converge solution, Table 1.

$u, v \quad$ Viscous-layer velocity components in directions of surface and wake streamline tangent and normal, respectively, (4.1-4.3).

$\tilde{v} \quad$ Scaled viscous-layer normal velocity component, (4.1).

$\mathcal{W} \quad$ Reference wake streamline, Figure 1.

$x, y \quad$ Airfoil frame coordinates, Figure 1 .

$x_{\text {sep }} \quad x$-location of separation point, Figure 6 .

Greek

$\beta \quad$ Pressure gradient parameter, (4.14).

$\gamma \quad$ Fluid specific heat ratio, (3.2).

$\gamma_{T} \quad$ Longitudinal intermittancy factor, (4.4).

$\delta \quad$ Displacement thickness, (4.8).

$\hat{\epsilon} \quad$ Convergence tolerance, (5.2).

$\bar{\varepsilon} \quad$ Ratio of turbulent to molecular viscosity coefficients, (4.13).

$\Theta \quad$ Cascade stagger angle, Figure 1.

$\theta \quad$ Momentum thickness, (4.9).

$\hat{\theta} \quad$ Viscous-layer local to edge temperature ratio, (4.15).

$\kappa \quad$ Wake streamline curvature, (3.5).

$\mu, \mu_{T} \quad$ Molecular and effective turbulent viscosities, (4.4). 
$\xi, \eta \quad$ Cascade axial and "circumferential" Cartesian coordinates, Figure 1; independent similarity variables, (4.10).

$\rho \quad$ Density, (3.2).

$\tau_{w} \quad$ Surface shear stress, e.g. Figure 4(c).

$\Phi \quad$ Velocity potential for inviscid flow, (3.1).

$\psi \quad$ Viscous-layer stream function, (4.11).

$\Omega \quad$ Flow angle measured from axial direction, positive counter-clockwise, Figure 1.

$\omega \quad$ Relaxation factor, (5.1).

$\bar{\omega} \quad$ Total pressure loss parameter, Figure 6 .

$\underline{\text { Subscripts }}$

$e \quad$ Viscous-layer edge value, (4.3).

$I, V \quad$ Inviscid, viscous values, (5.1).

$i \quad$ Index of solution station, (5.2).

$W, \mathcal{W}$ Wake value, (3.5), (4.22), (4.21).

TE Trailing-edge value, (4.6), (4.7).

$\mp \infty \quad$ Far upstream/downstream freestream value of variable, Figure 1.

$\underline{\text { Superscripts }}$

$n \quad$ Global iteration count, (5.1).

+, - Upper, lower viscous layer, (4.19), (4.22). 


\section{List of Figures}

Figure 1. Two-dimensional compressor cascade.

Figure 2. Semi-Inverse Iteration Procedure.

Figure 3. Streamline H-mesh for the EGV cascade.

Figure 4. Inviscid and IVI solutions for EGV cascade at $R e=10^{5}$ and $10^{6}$ : (a) pressure coefficient; (b) displacement thickness; (c) surface shear stress.

Figure 5. Comparison of IVI and Navier-Stokes solutions for the EGV cascade at $R e=10^{6}$ : (a) pressure coefficient; (b) displacement thickness; (c) surface shear stress.

Figure 6. Predictions for the EGV cascade for a range of inlet flow angles: (a) loss parameter; (b) exit flow angle; (c) separation point location.

Figure 7. Trailing-edge streamline patterns for the EGV cascade: (a) $\Omega_{-\infty}=36$ deg; (b) $\Omega_{-\infty}=45 \mathrm{deg}$; (c) $\Omega_{-\infty}=54 \mathrm{deg}$.

Figure 8. Streamline H-mesh for the HSC cascade.

Figure 9. Inviscid and IVI solutions for the HSC cascade at $R e=10^{5}$ and $10^{6}$ :

(a) pressure coefficient; (b) displacement thickness; (c) surface shear stress.

Figure 10. Comparison of IVI and Navier-Stokes solutions for the HSC cascade at $R e=10^{6}$ : (a) pressure coefficient; (b) displacement thickness; (c) surface shear stress.

Figure 11. Streamline H-mesh for the turbine cascade.

Figure 12. Results for turbine cascade: (a) comparison of predicted and measured Mach number distributions; (b) predicted displacement thickness distribution;

(c) predicted surface shear-stress distribution.

Figure 13. Convergence history for the EGV cascade at $R e=10^{6}$ and $\Omega_{-\infty}=40$ deg: (a) total pressure loss parameter; (b) exit flow angle. 


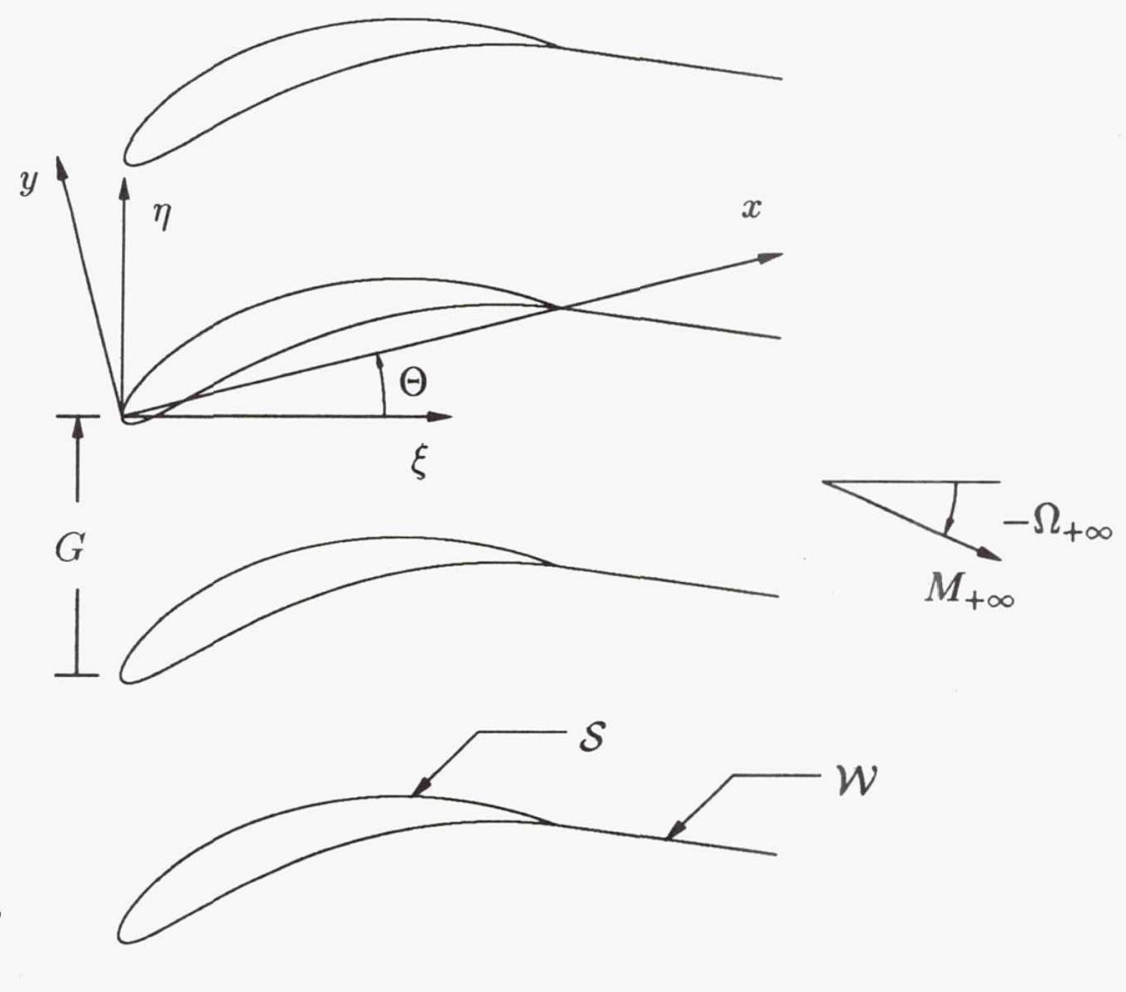

Figure 1: Two-dimensional compressor cascade. 


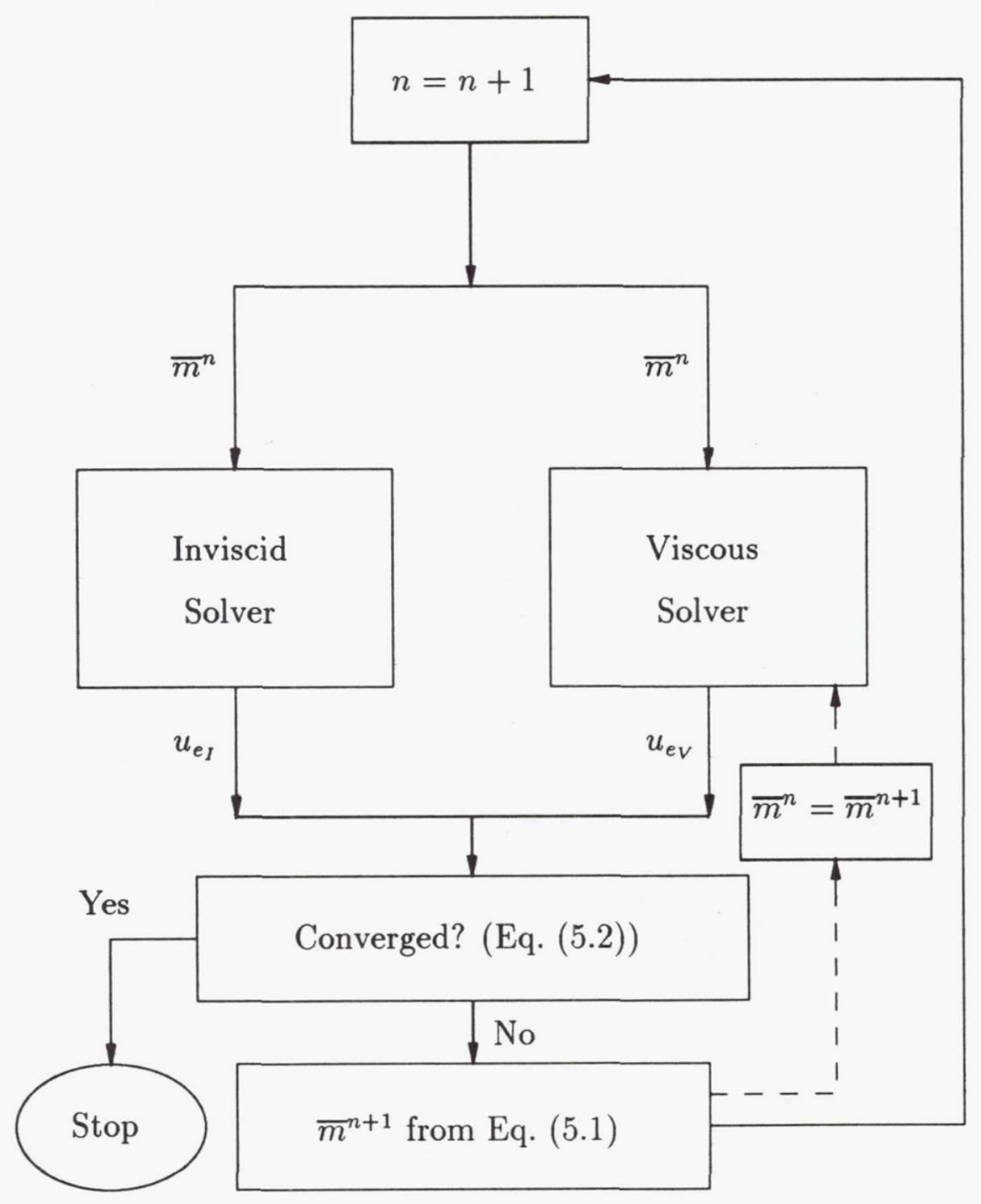

Figure 2: Semi-Inverse Iteration Procedure. 


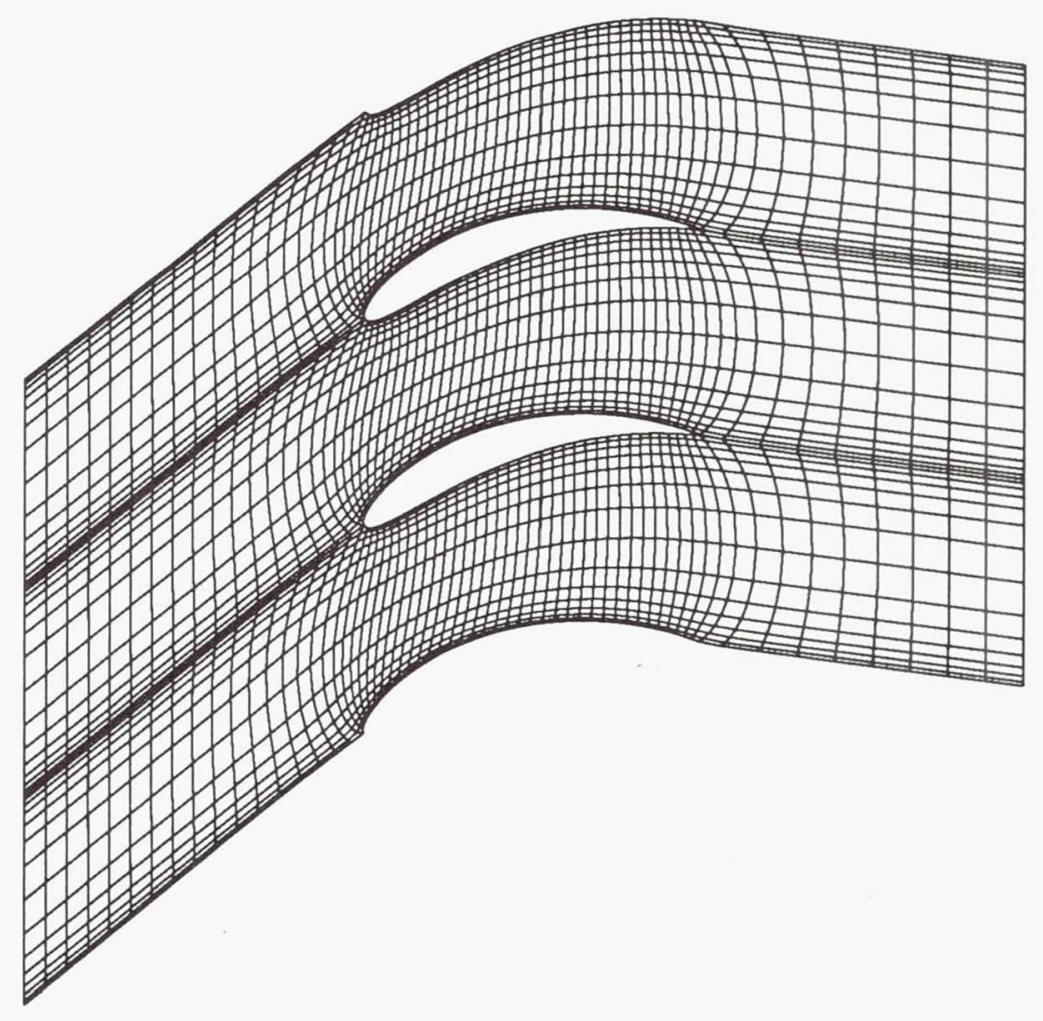

Figure 3: Streamline H-mesh for the EGV cascade. 


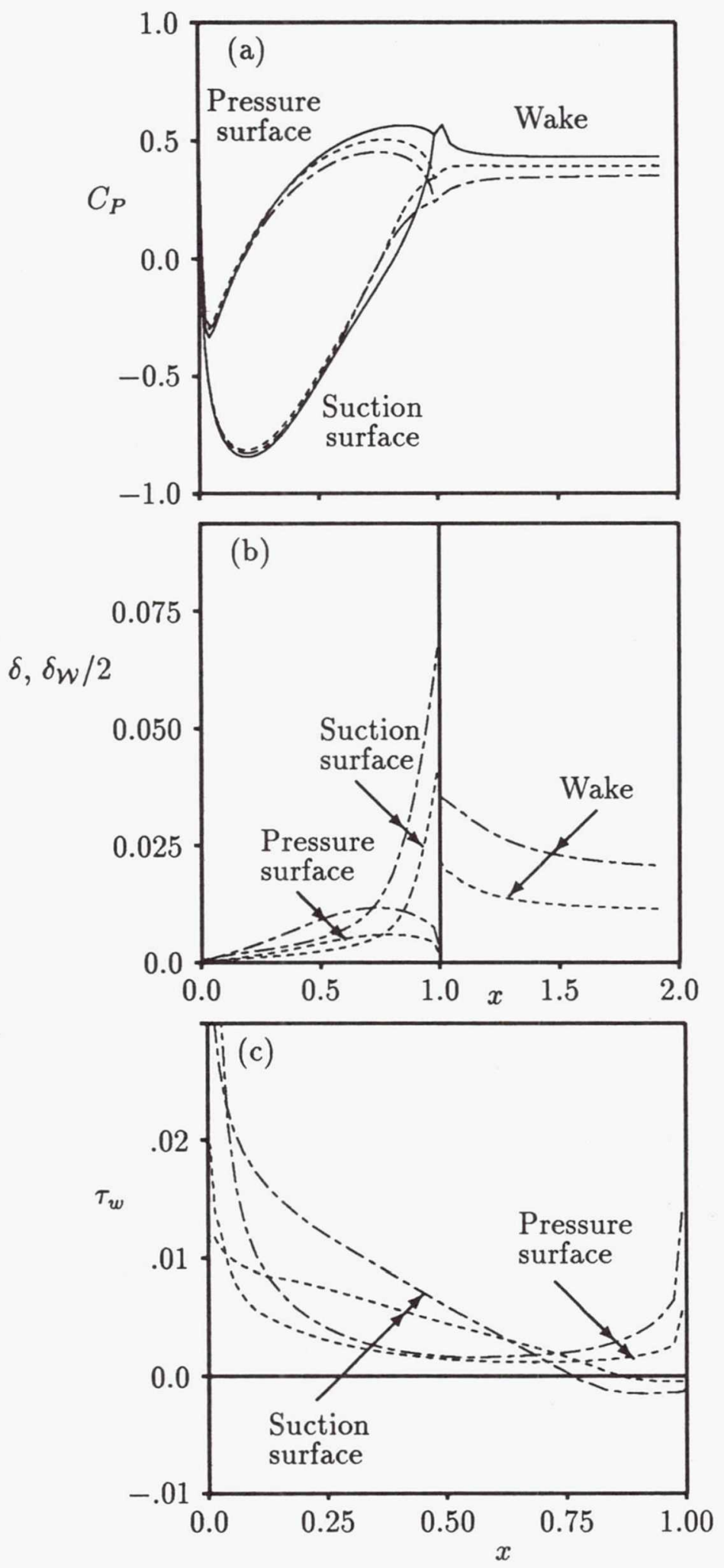

Figure 4: Inviscid (—) and IVI solutions for EGV cascade at $R e=10^{5}\left(----{ }_{-}\right)$and $10^{6}$ (- . - - ): (a) pressure coefficient; (b) displacement thickness; (c) surface shear stress. 


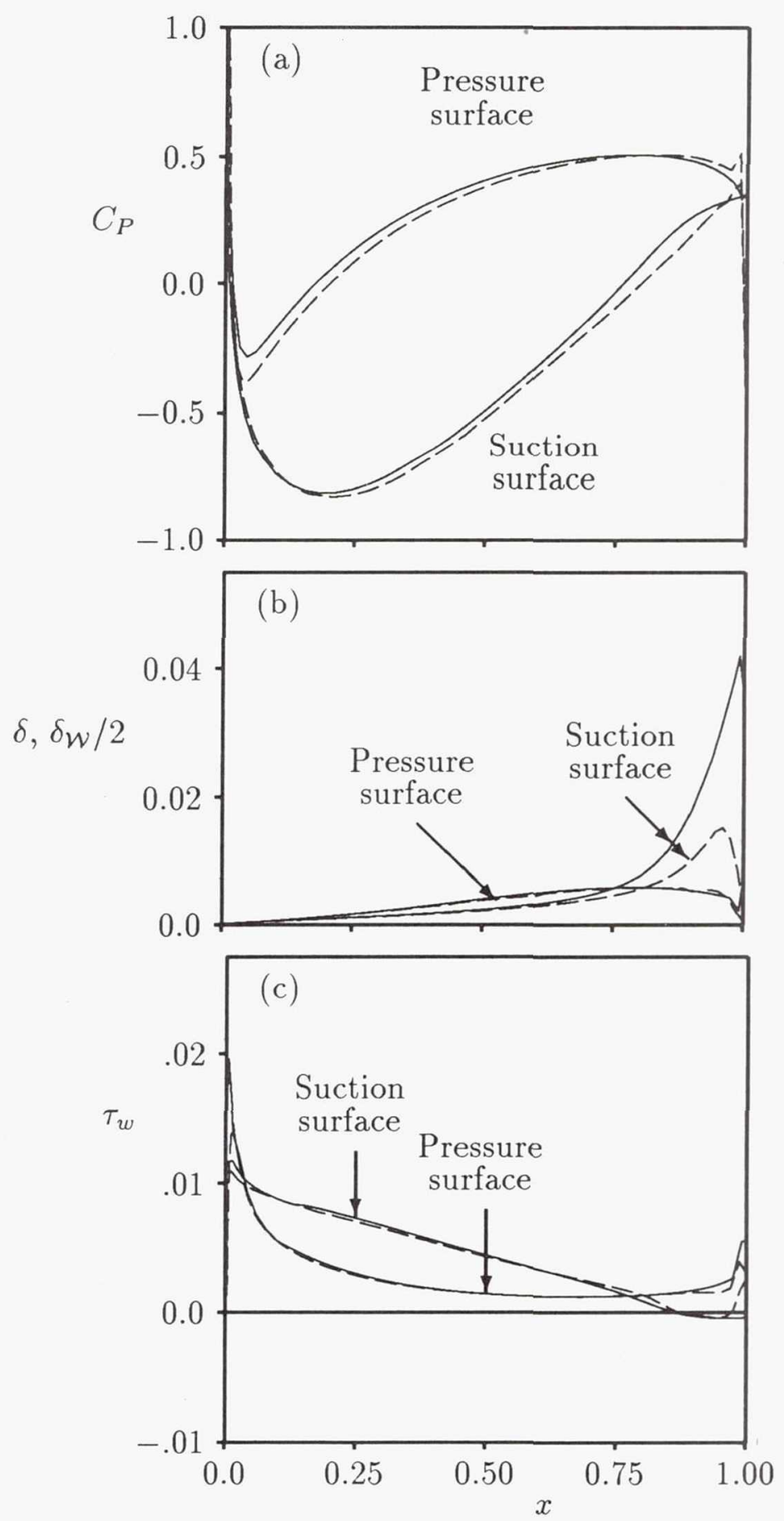

Figure 5: Comparison of IVI (__- ) and Navier-Stokes (- . - -) solutions for the EGV cascade at $R e=10^{6}$ : (a) pressure coefficient; (b) displacement thickness; (c) surface shear stress. 


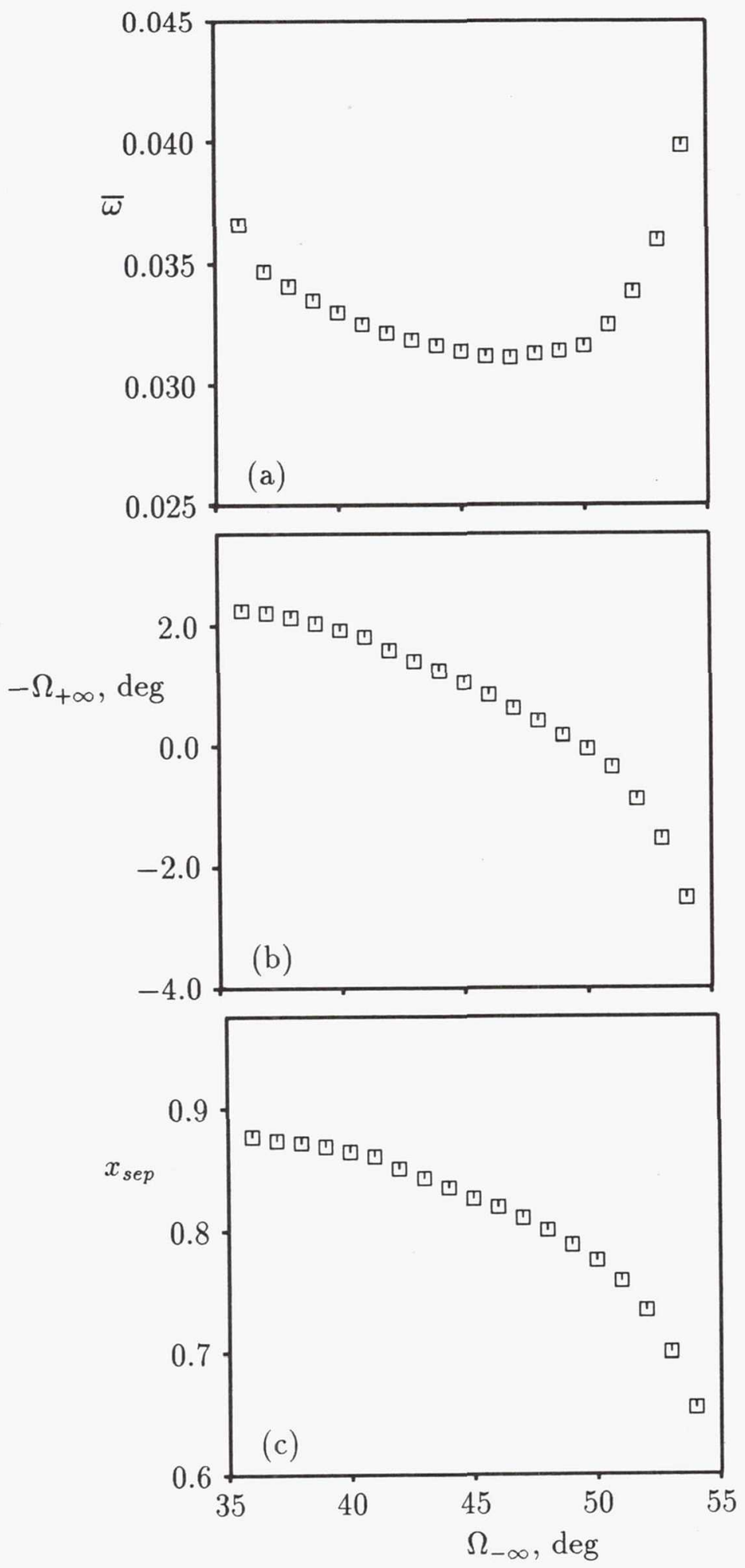

Figure 6: Predictions for the EGV cascade for a range of inlet flow angles: (a) loss parameter; (b) exit flow angle; (c) separation point location. 

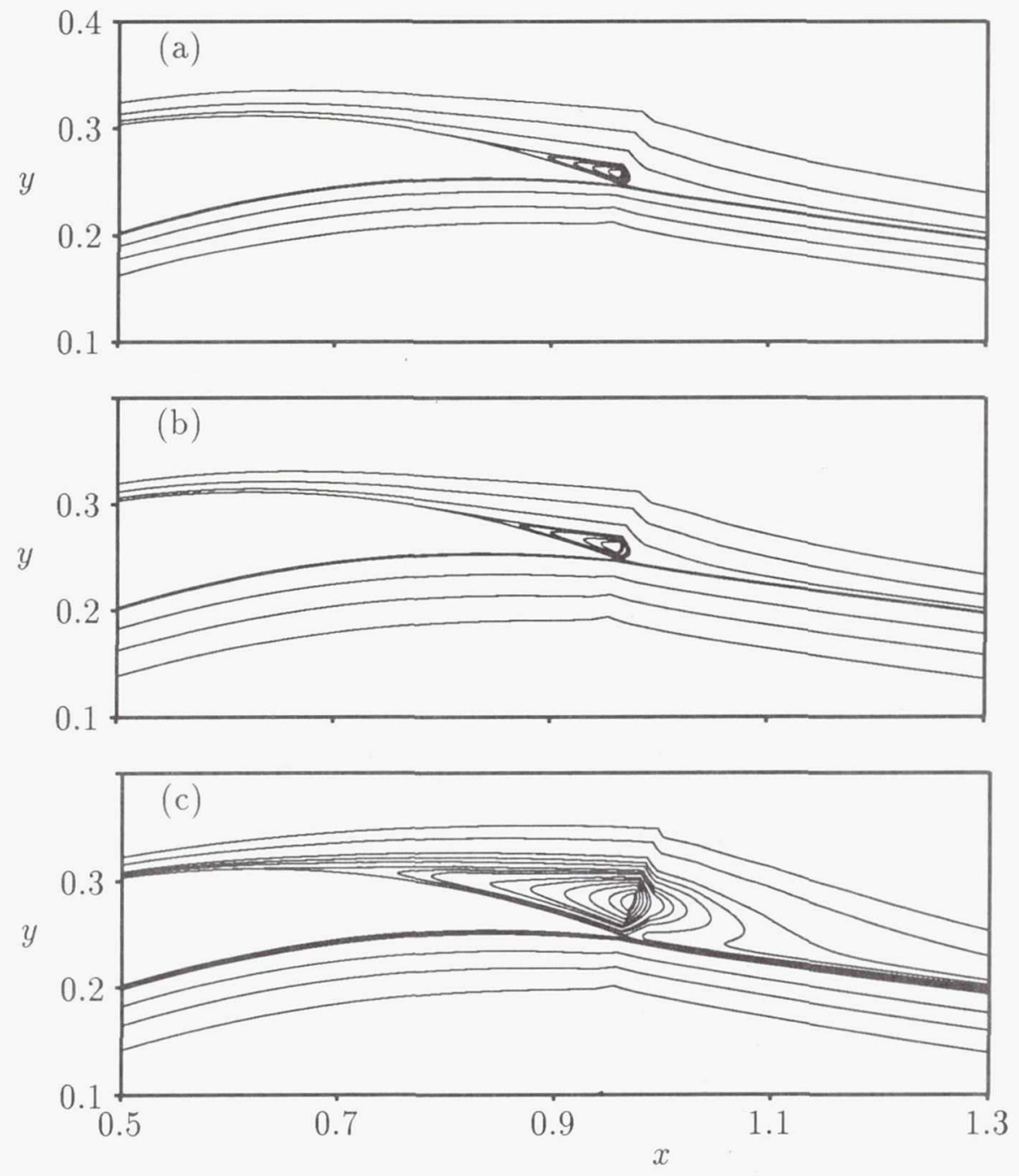

Figure 7: Trailing-edge streamline patterns for the EGV cascade: (a) $\Omega_{-\infty}=36 \mathrm{deg}$; (b) $\Omega_{-\infty}=45 \mathrm{deg} ;$ (c) $\Omega_{-\infty}=54 \mathrm{deg}$. 


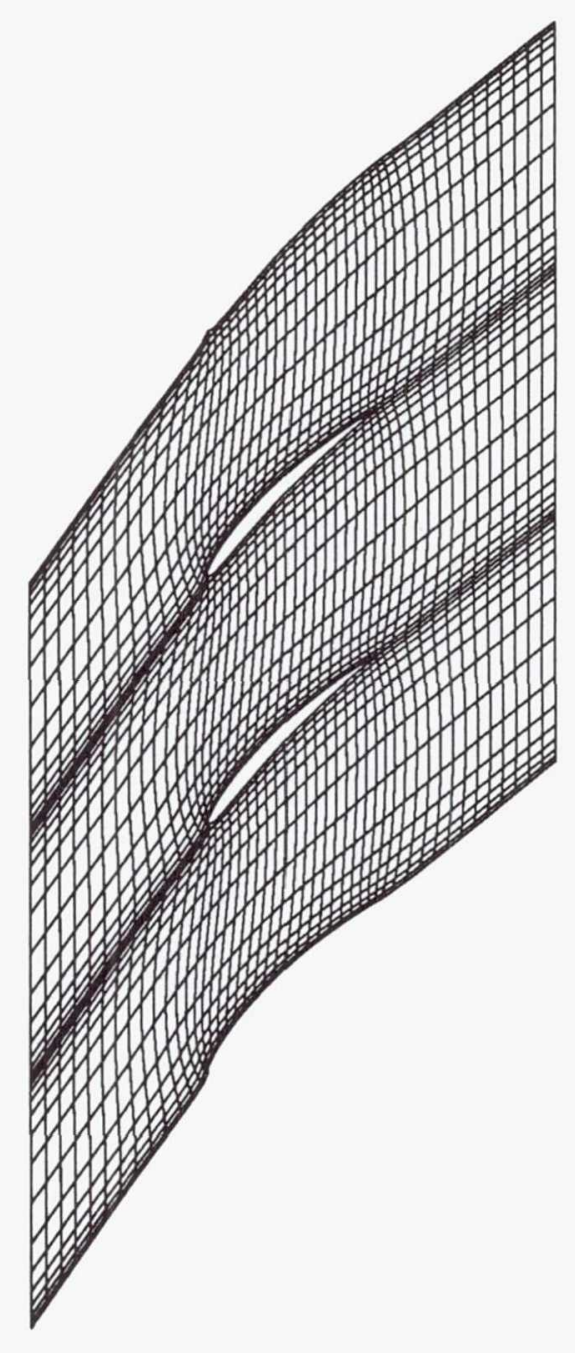

Figure 8: Streamline H-mesh for the HSC cascade. 


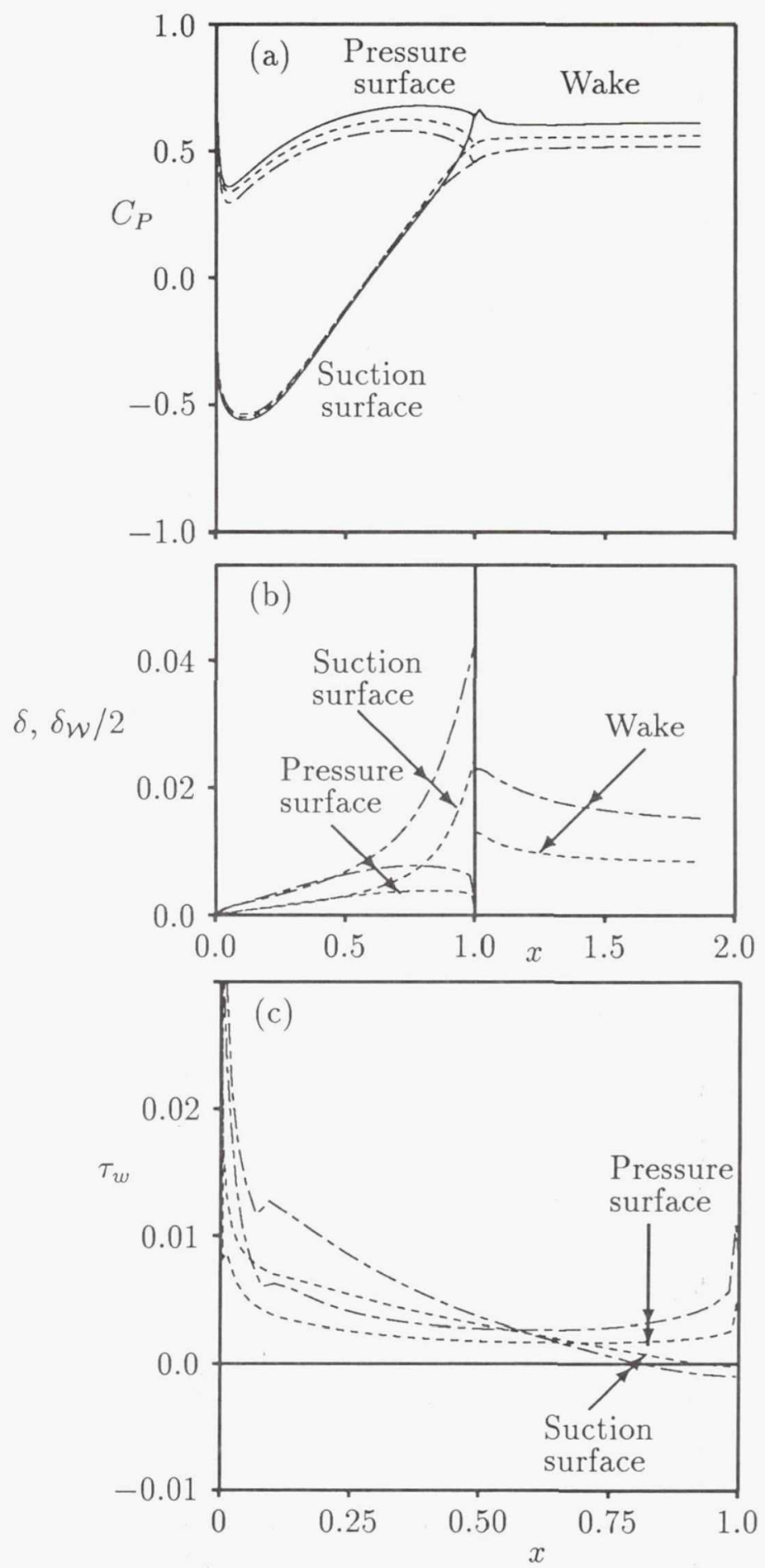

Figure 9: Inviscid (- - ) and IVI solutions for the HSC cascade at $R e=10^{5}\left(-{ }_{-}-{ }_{-}\right)$ and $10^{6}$ (...): (a) pressure coefficient; (b) displacement thickness; (c) surface shear stress. 

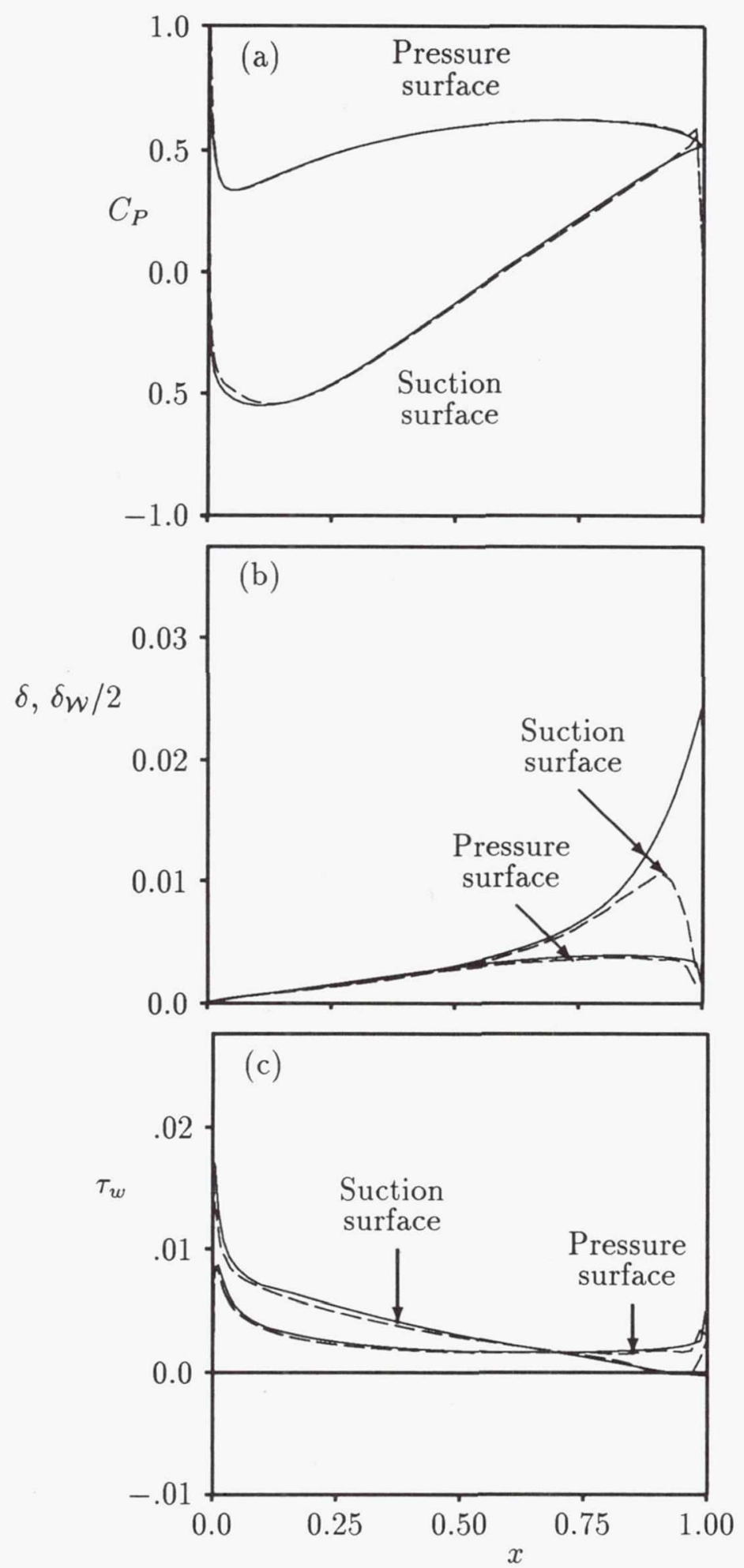

Figure 10: Comparison of IVI (__ ) and Navier-Stokes (- - - ) solutions for the HSC cascade at $R e=10^{6}$ : (a) pressure coefficient; (b) displacement thickness; (c) surface shear stress. 


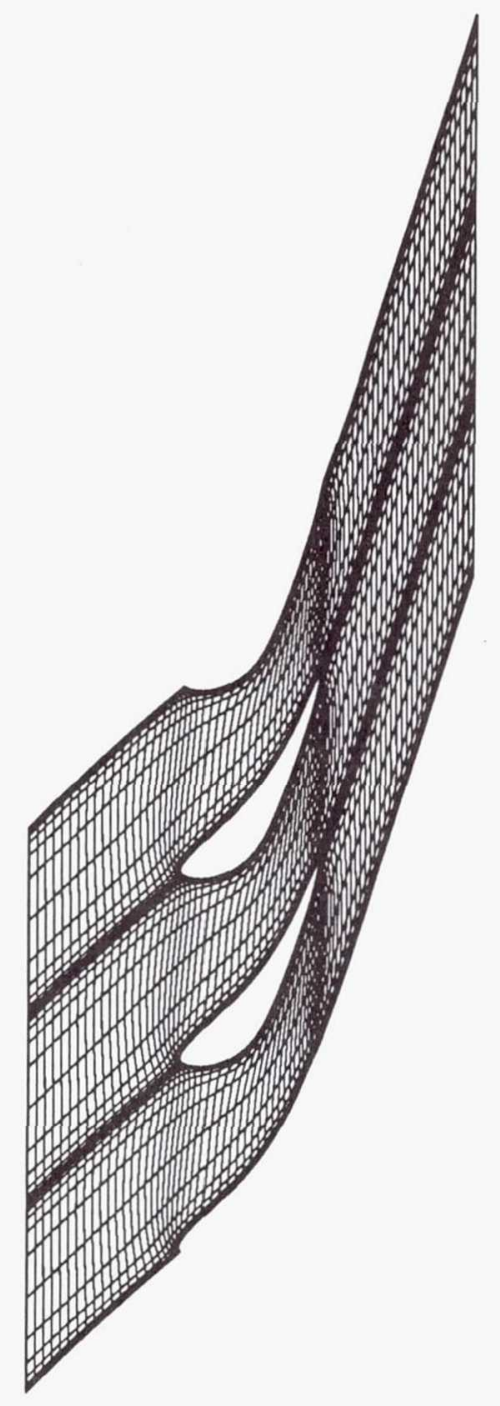

Figure 11: Streamline H-mesh for the turbine cascade. 

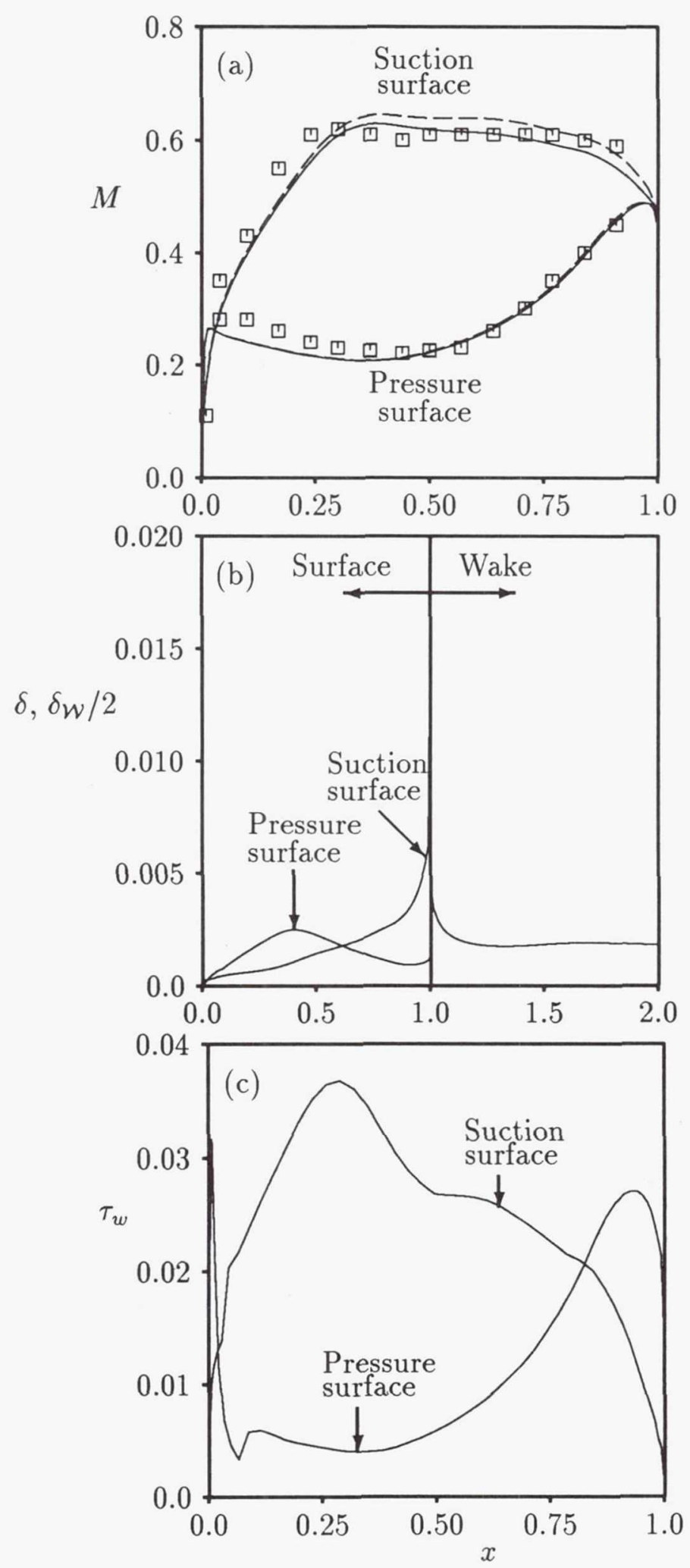

Figure 12: Results for turbine cascade: (a) comparison of predicted and measured Mach number distributions: (—— IVI; (- - - ) inviscid; symbols: experimental measurement; (b) predicted displacement thickness distribution; (c) predicted surface shear-stress distribution. 


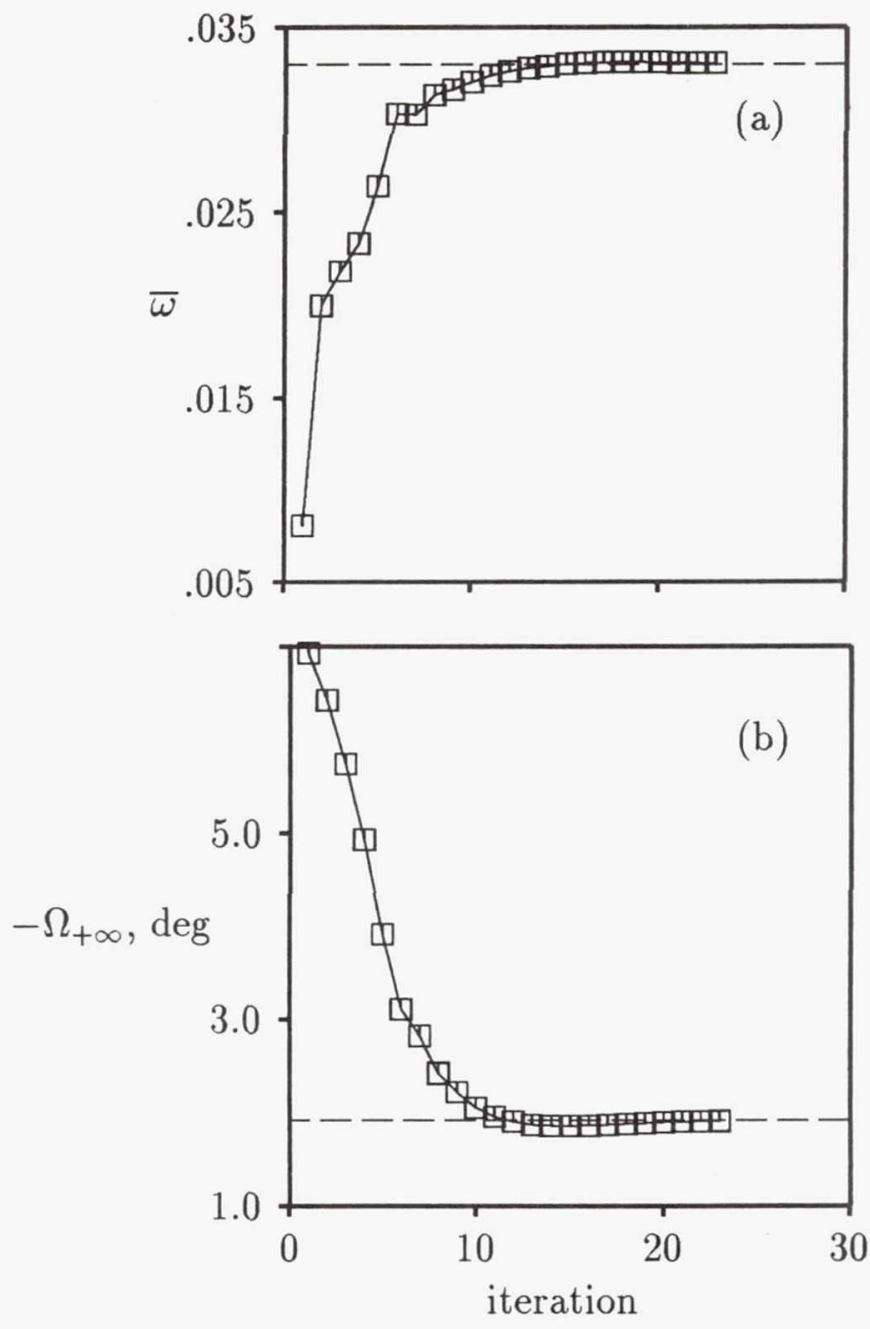

Figure 13: Convergence history for the EGV cascade at $R e=10^{6}$ and $\Omega_{-\infty}=40$ deg: (a) total pressure loss parameter; (b) exit flow angle. 


\section{A. Details of the Viscous-Layer Solution Procedure}

The viscous-layer equations are solved numerically using an implicit finite-difference approach, which is described in this Appendix. The finite-difference approximations used to discretize the governing equations and the quasi-linearization applied to the resulting system of nonlinear equations is discussed in $\S \mathrm{A} .1$. The recursion relations and the associated coefficients needed to solve the block-tridiagonal system of equations on the surface and along the wake are given in $\S$ A.2 and $\S$ A.3, respectively.

\section{A.1 Finite-Difference Approximations}

The partial derivatives appearing in the governing viscous-layer equations, (4.12) and (4.13), are all first derivatives, except for the shear term appearing in the latter equation, which introduces a second derivative with respect to $\eta$. In the present analysis, first-order accurate backward differences are used to approximate $\partial / \partial \xi$ terms and second-order accurate central differences are used to approximate first and second partial derivatives with respect to $\eta$. In the description that follows the subscripts $i$ and $j$ are the mesh-point indices in the $\xi$ - and $\eta$-directions, respectively, so that the notation $(\cdot)_{i, j}$ refers to a quantity evaluated at the location $\left(\xi_{i}, \eta_{j}\right)$. For convenience, the $i$ index will only be used to denote quantities evaluated at $\xi$ locations other than $\xi_{i}$, so that if no $i$ index is given, the variable is evaluated at $\xi_{i}$, i.e., $(\cdot)_{j}$ refers to $\left(\xi_{i}, \eta_{j}\right)$. Surface viscous layers are discretized in the surface normal direction using $N J$ points and $2 N J-1 \stackrel{\text { def }}{=} N J 2$ points are used across the wake. The index $j=1$ at blade surfaces $(\eta=0)$ and $j=N J$ at the outer edge of surface viscous layers $\left(\eta=\eta_{e}\right)$. In wakes $j=1$ at the lower edge $\left(\eta=\eta_{e}^{-}\right), j=N J$ along the wake reference streamline $(\eta=0)$ and $j=N J 2$ at the upper edge of the wake viscous layer $\left(\eta=\eta_{e}^{+}\right)$.

Both the direct and inverse modes are used on the blade surface, while only the inverse mode is used in the wake; both modes are described below. The procedure used to solve for the surface and the wake viscous layers is similar, however, in the latter case, the boundary conditions are applied at three different locations, requiring a modification to the surface technique, as detailed in $§$ A.3.

First derivatives of the dependent variables are written as

$$
\left(\frac{\partial F}{\partial \xi}\right)_{j} \approx \frac{F_{j}-F_{i-1, j}}{\Delta \xi}
$$

and

$$
\left(\frac{\partial F}{\partial \eta}\right)_{j} \approx \frac{F_{j+1}-F_{j-1}}{\Delta \eta_{j+1}+\Delta \eta_{j}},
$$

where

$$
\begin{aligned}
\Delta \xi & =\xi_{i}-\xi_{i-1} \\
\Delta \eta_{j+1} & =\eta_{j+1}-\eta_{j}
\end{aligned}
$$

and

$$
\Delta \eta_{j}=\eta_{j}-\eta_{j-1}
$$


The second derivative with respect to $\eta$ is discretized as follows:

$$
\frac{\partial}{\partial \eta}\left(\ell \bar{\varepsilon} \frac{\partial F}{\partial \eta}\right)_{j} \approx \frac{2}{\Delta \eta_{j}+\Delta \eta_{j-1}}\left(\ell \bar{\varepsilon}_{j+1 / 2} \frac{F_{j+1}-F_{j}}{\Delta \eta_{j}}-\ell \bar{\varepsilon}_{j-1 / 2} \frac{F_{j}-F_{j-1}}{\Delta \eta_{j-1}}\right),
$$

where, e.g., $\ell \bar{\varepsilon}_{j+1 / 2}=\left(\ell \bar{\varepsilon}_{j+1}+\ell \bar{\varepsilon}_{j}\right) / 2$. If, as in all of the calculations presented here, $\left|1-\Delta \eta_{j} / \Delta \eta_{j-1}\right| \ll 1$, the $\eta$-derivatives remain formally second-order accurate.

Before discretizing the equations all variables are written in "delta" form, e.g., $F_{j}=$ $F_{j}^{g}+\delta F_{j}$, where the superscript $g$ denotes the value of $F_{j}$ from the previous iteration or an initial guess. Thus, rather than solve for the variables themselves, the changes in the variables (e.g., $\delta F_{j}$ ) are solved for. Following this procedure for the continuity equation, (4.12), leads to the expression

$$
\delta f_{j}-\delta f_{j-1}+P_{j}\left(\delta F_{j}+\delta F_{j-1}\right)=Q_{j},
$$

where

$$
\begin{aligned}
P_{j} & =-\Delta \eta_{j} / 2, \\
Q_{j} & =-P_{j}\left(F_{j}+F_{j-1}\right)-f_{j}+f_{j-1} .
\end{aligned}
$$

In this equation and those that follow, the superscript $g$ has been dropped for convenience. The momentum equation can be written, after discretizing and letting $a_{1}=1 /\left(\Delta \eta_{j}+\Delta \eta_{j+1}\right)$ and $a_{2}=2 \xi_{i} / \Delta \xi$, in the form

$$
A_{j} \delta F_{j-1}+B_{j} \delta F_{j}+C_{j} \delta F_{j+1}+D_{j} \delta f_{j}=E_{1 j} \delta \beta+E_{2 j},
$$

where

$$
\begin{aligned}
& A_{j}=a_{1}\left[\frac{2(\ell \bar{\varepsilon})_{j-1 / 2}}{\Delta \eta_{j}}-b_{1}\left\{f_{j}+a_{2}\left(f_{j}-f_{i-1, j}\right)\right\}\right], \\
& B_{j}=-2 a_{1}\left(\frac{(\ell \bar{\varepsilon})_{j+1 / 2}}{\Delta \eta_{j+1}}+\frac{(\ell \bar{\varepsilon})_{j-1 / 2}}{\Delta \eta_{j-1}}\right)-b_{1}\left[a_{2}\left(2 F_{j}-F_{i-1, j}\right)+2 \beta F_{j}\right], \\
& C_{j}=a_{1}\left[\frac{2(\ell \bar{\varepsilon})_{j+1 / 2}}{\Delta \eta_{j+1}}+b_{1}\left\{f_{j}+a_{2}\left(f_{j}-f_{i-1, j}\right)\right\}\right], \\
& D_{j}=b_{1} a_{1}\left(1+a_{2}\right)\left(F_{j+1}-F_{j-1}\right), \\
& E_{1 j}=b_{1} F_{j}^{2}-\hat{\theta}_{j}
\end{aligned}
$$

and

$$
\begin{aligned}
E_{2 j}= & -2 a_{1}\left[\frac{(\ell \bar{\varepsilon})_{j+1 / 2}}{\Delta \eta_{j+1}} F_{j+1}-\left(\frac{(\ell \bar{\varepsilon})_{j+1 / 2}}{\Delta \eta_{j+1}}+\frac{(\ell \bar{\varepsilon})_{j-1 / 2}}{\Delta \eta_{j}}\right) F_{j}+\frac{(\ell \bar{\varepsilon})_{j-1 / 2}}{\Delta \eta_{j}} F_{j-1}\right] \\
& +b_{1} a_{2} F_{j}\left(F_{j}-F_{i-1, j}\right)-b_{1} a_{1}\left[f_{j}+a_{2}\left(f_{j}-f_{i-1, j}\right)\right]\left(F_{j+1}-F_{j-1}\right)+\left(b_{1} F_{j}^{2}-\hat{\theta}_{j}\right) \beta .
\end{aligned}
$$


The quantity $b_{1}$ controls the FLARE approximation [34] used in separated flow regions; when $F \leq 0$ at any of the points $j-1, j$ or $j+1$ at either $i$ or $i-1$ (except the wall point at $j=1), b_{1}$ is set to zero, otherwise $b_{1}=1$. Note that the term $E_{1} \delta \beta$ is only included in the inverse mode, where $\beta$ is one of the unknowns; in the direct mode $\delta \beta=0$.

In deriving the discretized equations, the nonlinear terms, which have the generic form $U V$, are expanded using the "delta" form of the variables as follows:

$$
U V=\left(U^{g}+\delta U\right)\left(V^{g}+\delta V\right)=U^{g} V^{g}+U^{g} \delta V+V^{g} \delta U+\delta U \delta V
$$

The governing equations are quasi-linearized by neglecting $\mathcal{O}\left(\delta^{2}\right)$ terms (i.e., $\left.\delta U \delta V\right)$. The truncation error associated with the quasi-linearization is eliminated by performing multiple iterations at each solution station.

The algebraic system of equations resulting from the discretization and quasi-linearization of the original partial differential equations is block-tridiagonal in form, allowing standard techniques to be used for its solution. The surface and wake solution procedures are similar, although the wake analysis is more complicated because of the differences between the boundary conditions used for each procedure.

\section{A.2 Surface Procedure}

The system of equations applicable to the surface viscous layers is solved using the blocktridiagonal inversion procedure described below. The recursion relations for the unknown variables $\delta F_{j}$ and $\delta f_{j}$ are written as follows:

$$
\delta F_{j}=R_{j}-S_{j} \delta \beta-T_{j} \delta F_{j+1}
$$

and

$$
\delta f_{j}=L_{j}-M_{j} \delta \beta-N_{j} \delta F_{j+1} .
$$

In the direct mode, where $\beta$ is known, the coefficients $S_{j}$ and $M_{j}$ are set equal to zero.

Substituting for $\delta F_{j-1}$ and $\delta f_{j-1}$ in the continuity equation (A.4) using Eqs. (A.9) and (A.10), solving for $\delta f_{j}$ and using this expression and Eq. (A.9) to eliminate $\delta F_{j-1}$ yields, after collecting terms, the following expressions for $R_{j}, S_{j}$ and $T_{j}$ :

$$
\begin{aligned}
& R_{j}=d_{1}\left[A_{j} R_{j-1}+D_{j}\left(Q_{j}+L_{j-1}-P_{j} R_{j-1}\right)-E_{2 j}\right], \\
& S_{j}=d_{1}\left[A_{j} S_{j-1}+D_{j}\left(M_{j-1}-P_{j} S_{j-1}\right)+E_{1 j}\right],
\end{aligned}
$$

and

$$
T_{j}=-d_{1} C_{j}
$$

where

$$
d_{1}=1 /\left[A_{j} T_{j-1}-B_{j}+D_{j}\left(N_{j-1}+P_{j}\left\{1-T_{j-1}\right\}\right)\right] .
$$


Returning to the expression for $\delta f_{j}$ obtained by manipulating the continuity equation as described in the preceeding paragraph and substituting for $\delta F_{j}$ using Eq. (A.9) produces the following expressions for the coefficients appearing in Eq. (A.10):

$$
\begin{aligned}
L_{j} & =Q_{j}+L_{j-1}-P_{j} R_{j-1}-R_{j}\left[N_{j-1}+P_{j}\left(1-T_{j-1}\right)\right], \\
M_{j} & =M_{j-1}-P_{j} S_{j-1}-S_{j}\left[N_{j-1}+P_{j}\left(1-T_{j-1}\right)\right]
\end{aligned}
$$

and

$$
N_{j}=-T_{j}\left[N_{j-1}+P_{j}\left(1-T_{j-1}\right)\right] .
$$

The recursion relation coefficients are obtained using Eqs. (A.11)-(A.13), sweeping from $j=2$ to $j=N J-1$. The values of the coefficients at $j=1$ are needed; they are obtained from the boundary conditions for $f$ and $F$, Eqs. (4.17) and (4.18). Since the values of $F$ and $f$ are known at $j=1$, we set $\delta F_{j=1}=\delta f_{j=1} \equiv 0$, which gives

$$
R_{1}=S_{1}=T_{1} \equiv 0
$$

and

$$
L_{1}=M_{1}=N_{1} \equiv 0 \text {. }
$$

The solution for $\delta F_{j}$ and $\delta f_{j}$ is obtained by sweeping the recursion relations, Eqs. (A.9) and (A.10), from $j=N J-1$ to $j=2$. This requires knowledge of the values of $\delta F_{N J}$, $\delta f_{N J}$ and $\delta \beta$. The quantity $\delta F_{N J}=0$, since $F_{N J}=1$ (Eq. (4.19)). The technique used to determine the value of $\delta f_{N J}$ depends on whether the direct or inverse mode is being used, as described below.

In direct mode the value of $\beta$ is known, hence $\delta \beta=0$. Writing the continuity equation (A.4) at $j=N J$ and substituting Eqs. (A.9) and (A.10) evaluated at $j=N J-1$ yields

$$
\delta f_{N J}=Q_{N J}+L_{N J-1}-P_{N J} R_{N J-1} .
$$

In inverse mode the value of $\delta f_{N J}$ is prescribed using Eq. (4.20), which is rewritten in "delta" form as

$$
\delta f_{N J}=h+\eta_{N J}-\frac{\bar{m}}{\sqrt{2 \xi}}-f_{N J} .
$$

Combining this relation, Eq. (A.4) written at $j=N J$ and Eqs. (A.9) and (A.10) evaluated at $j=N J-1$ yields the following solution for $\delta \beta$ :

$$
\delta \beta=\frac{Q_{N J}-\delta f_{N J}+L_{N J-1}-P_{N J} R_{N J-1}}{M_{N J-1}-P_{N J} S_{N J-1}} .
$$

With all variables known at $j=N J$, Eqs. (A.9) and (A.10) are swept from $j=N J-1$ to $j=2$ to solve for $\delta F_{j}$ and $\delta f_{j}$. Finally, the values of $F_{j}$ and $f_{j}$ are obtained from the relations

$$
F_{j}=F_{j}^{g}+\delta F_{j}
$$


and

$$
f_{j}=f_{j}^{g}+\delta f_{j}
$$

and the updated value of $\beta$ from

$$
\beta=\beta^{g}+\delta \beta
$$

Because the viscous-layer equations are nonlinear, this solution procedure is applied iteratively at each $i$-station until the solution at $\xi_{i}$ converges. Convergence occurs when the maximum magnitude of the residuals of the continuity and momentum equations, $\max _{2 \leq j \leq N J}\left|Q_{j}\right|$ and $\max _{2 \leq j \leq N J-1}\left|E_{2 j}\right|$, respectively, are less than a specified tolerance, which we usually set to $5 \times 10^{-4}$. Convergence is typically obtained in from two to five iterations.

\section{A.3 Wake Procedure}

The solution procedure used for the wake viscous layers is very similar to that used for the surface layers. However, modifications must be made to account for the application of two boundary conditions at the upper and lower edges of the viscous layer (Eq. (4.19)) and a third boundary condition along the wake reference streamline (Eq. (4.17)). In addition, since the inverse mode is used in the wake, a jump in $f$ from $j=1$ (at $\eta=\eta_{e}^{-}$) to $j=N J 2$ (at $\eta=\eta_{e}^{+}$) is imposed (Eq. (4.22)).

An additional term is appended to the recursion relations used for the surface viscouslayer analysis; the wake recursion relations are given by:

$$
\delta F_{j}=R_{j}-S_{j} \delta \beta-T_{j} \delta F_{j+1}-U_{j} \delta f_{N J 2}
$$

and

$$
\delta f_{j}=L_{j}-M_{j} \delta \beta-N_{j} \delta F_{j+1}-H_{j} \delta f_{N J 2} .
$$

Using the same approach employed for the surface analysis we obtain the coefficients in

Eq. (A.22). The expressions for $R_{j}, S_{j}$ and $T_{j}$ are identical to those given in Eq. (A.11), and the value of $U_{j}$ is given by

$$
U_{j}=d_{1}\left[A_{j} U_{j-1}+D_{j}\left(H_{j-1}-P_{j} U_{j-1}\right)\right],
$$

where $d_{1}$ is given by Eq. (A.12). The coefficients $L_{j}, M_{j}$ and $N_{j}$ appearing in Eq. (A.23) are defined in Eq. (A.13), and the additional coefficient $H_{j}$ is given by

$$
H_{j}=H_{j-1}-P_{j} U_{j-1}-U_{j}\left(N_{j-1}+P_{j}\left\{1-T_{j-1}\right\}\right) .
$$

At the lower edge of the viscous layer $\left(\eta=\eta_{e}^{-}, j=1\right) F_{1}=1$, therefore $\delta F_{1}=0$, yielding

$$
R_{1}=S_{1}=T_{1}=U_{1} \equiv 0 \text {. }
$$

The jump condition for $f$ is rewritten in delta form as

$$
\delta f_{N J 2}-\delta f_{1}=f_{1}-f_{N J 2}+h+\left(\eta_{e}^{+}-\eta_{e}^{-}\right)-\frac{\bar{m}_{\mathcal{W}}}{\sqrt{2 \xi}},
$$


which can be rearranged in the form of Eq. (A.23) evaluated at $j=1$ to give

$$
\begin{aligned}
L_{1} & =f_{N J 2}-f_{1}-h-\left(\eta_{e}^{+}-\eta_{e}^{-}\right)+\frac{\bar{m}_{\mathcal{W}}}{\sqrt{2 \xi}}, \\
M_{1} & =N_{1} \equiv 0
\end{aligned}
$$

and

$$
H_{1}=-1 \text {. }
$$

With the recursion relation coefficients determined at $j=1$, the coefficients at the remaining $j$-locations can be determined.

The remaining boundary conditions, namely

$$
\delta f_{N J}=0
$$

and

$$
\delta F_{N J 2}=0,
$$

must be applied and the value of $\delta \beta$ obtained before the system of equations can be inverted to obtain $\delta F_{j}$ and $\delta f_{j}$. To accomplish this, the complete system of equations given by the recursion relations, the remaining boundary conditions and the continuity equation applied at $j=N J 2$ (which has not yet been invoked) are written in matrix form as follows. At each point $j \in[1, N J 2-1]$ we can write the recursion relations in the form

$$
\bar{A}_{j} \delta \bar{U}_{j}+\bar{B}_{j} \delta \bar{U}_{j+1}+\bar{e}_{j} \delta \bar{V}=\bar{d}_{j},
$$

where

$$
\begin{aligned}
\bar{A}_{j} & =\left[\begin{array}{ll}
1 & 0 \\
0 & 1
\end{array}\right], \\
\bar{B}_{j} & =\left[\begin{array}{ll}
T_{j} & 0 \\
N_{j} & 0
\end{array}\right], \\
\bar{e}_{j} & =\left[\begin{array}{lll}
0 & U_{j} & S_{j} \\
0 & H_{j} & M_{j}
\end{array}\right], \\
\delta \bar{U}_{j} & =\left[\begin{array}{l}
\delta F_{j} \\
\delta f_{j}
\end{array}\right], \\
\delta \bar{V} & =\left[\begin{array}{c}
\delta F_{N J 2} \\
\delta f_{N J 2} \\
\delta \beta
\end{array}\right]
\end{aligned}
$$

and

$$
\bar{d}_{j}=\left[\begin{array}{l}
R_{j} \\
L_{j}
\end{array}\right] .
$$


The complete matrix system of equations to be inverted has the form

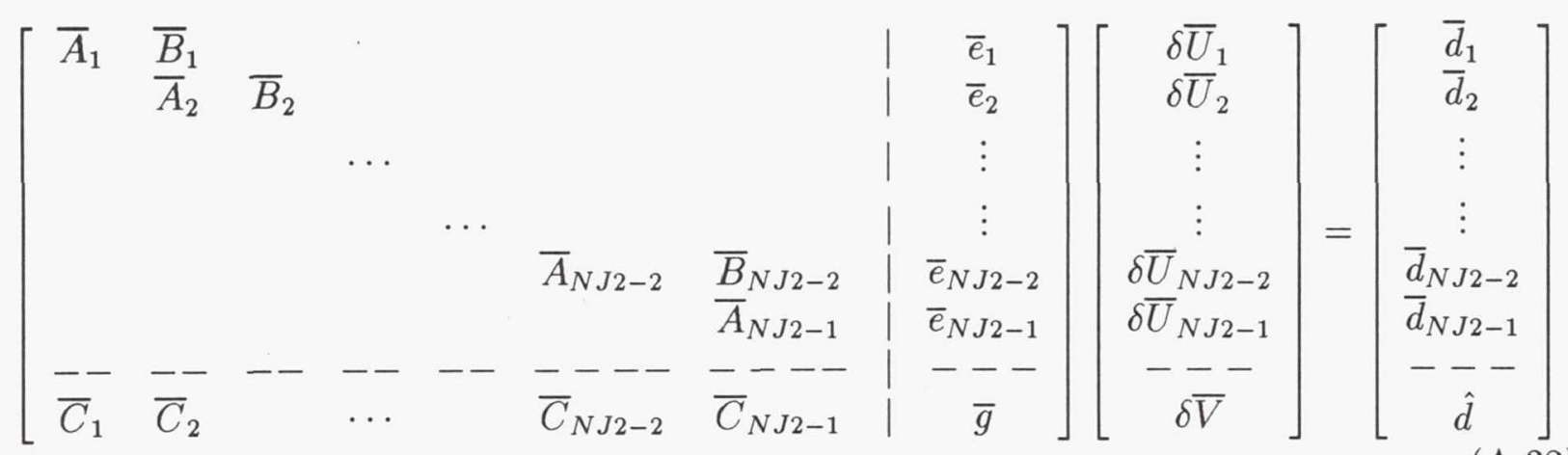

where

$$
\begin{aligned}
\bar{C}_{j} & =\left[\begin{array}{ll}
0 & 0 \\
0 & 0 \\
0 & 0
\end{array}\right] \text { for } 1 \leq j \leq N J 2-2, j \neq N J \\
\bar{C}_{N J} & =\left[\begin{array}{ll}
0 & 0 \\
0 & 1 \\
0 & 0
\end{array}\right], \\
\bar{C}_{N J 2-1} & =\left[\begin{array}{cc}
P_{N J 2} & -1 \\
0 & 0 \\
0 & 0
\end{array}\right] \\
\bar{g} & =\left[\begin{array}{ccc}
P_{N J 2} & 1 & 0 \\
0 & 0 & 0 \\
1 & 0 & 0
\end{array}\right]
\end{aligned}
$$

and

$$
\hat{d}=\left[\begin{array}{c}
Q_{N J 2} \\
0 \\
0
\end{array}\right] .
$$

Note that $\bar{e}_{N J 2-1}$ is modified from the standard form of $\bar{e}_{j}$ so that

$$
\bar{e}_{N J 2-1}=\left[\begin{array}{ccc}
T_{j} & U_{j} & S_{j} \\
N_{j} & H_{j} & M_{j}
\end{array}\right]_{j=N J 2-1} .
$$

Eq. (A.32) can be rewritten as a partitioned matrix of the form

$$
\left(\begin{array}{c|c}
\Gamma & \bar{e} \\
-\overline{-}- & --- \\
\bar{C}^{T} & \bar{g}
\end{array}\right)\left(\begin{array}{c}
\delta \bar{U} \\
-\bar{\delta} \\
\delta \bar{V}
\end{array}\right)=\left(\begin{array}{c}
\bar{d} \\
-\overline{\hat{d}}
\end{array}\right) .
$$

The submatrix $\Gamma$ is an upper block-bidiagonal matrix - the only nonzero terms are the nonzero entries appearing in the $2 \times 2$ blocks denoted by $\bar{A}$ and $\bar{B}$ in Eq. (A.32). The row 
vector $\bar{C}^{T}$ consists of the $3 \times 1$ vector elements $\bar{C}$, and represents the continuity equation written between $N J 2$ and $N J 2-1$, and the boundary conditions $\delta f_{N J}=0$ and $\delta F_{N J 2}=0$, respectively.

Expanding the partitioned matrix and combining the two resulting equations yields

$$
\delta \bar{V}=\left(\bar{g}-\bar{C}^{T} \Gamma^{-1} \bar{e}\right)^{-1}\left(\hat{d}-\bar{C}^{T} \Gamma^{-1} \bar{d}\right) .
$$

Letting

$$
\Gamma \bar{X}=\bar{d}
$$

and

$$
\Gamma \bar{Y}=\bar{e}
$$

allows Eq. (A.35) to be rewritten in the form

$$
\delta \bar{V}=\left(\bar{g}-\bar{C}^{T} \bar{Y}\right)^{-1}\left(\hat{d}-\bar{C}^{T} \bar{X}\right) .
$$

Equations (A.36) and (A.37) are easily inverted to solve for the elements of $\bar{X}$ and $\bar{Y}$ since $\Gamma$ is an upper block-bidiagonal matrix. Noting that $\bar{A}$ is the $2 \times 2$ identity matrix, the elements of $\bar{X}$ and $\bar{Y}$ are given by

$$
\begin{aligned}
\bar{X}_{N J 2-1} & =\bar{d}_{N J 2-1} \\
\bar{X}_{j} & =\bar{d}_{j}-\bar{B}_{j} \bar{X}_{j+1}, j=N J 2-2 \downarrow 1
\end{aligned}
$$

and

$$
\begin{aligned}
\bar{Y}_{N J 2-1} & =\bar{e}_{N J 2-1}, \\
\bar{Y}_{j} & =\bar{e}_{j}-\bar{B}_{j} \bar{Y}_{j+1}, j=N J 2-2 \downarrow 1 .
\end{aligned}
$$

The final solution for $\delta \bar{V}$ is obtained by carrying out the matrix multiplications for $\bar{C}^{T} \bar{X}$ and $\bar{C}^{T} \bar{Y}$, forming $\hat{d}-\bar{C}^{T} \bar{X}$ and $\bar{g}-\bar{C}^{T} \bar{Y}$, inverting the $3 \times 3$ matrix $\bar{g}-\bar{C}^{T} \bar{Y}$ and solving Eq. (A.38).

With the elements of $\delta \bar{V}$ known, the recursion relations, Eqs. (A.22) and (A.23), are applied to obtain the solution for $\delta F_{j}$ and $\delta f_{j}$ for $1 \leq j \leq N J 2-1$. Finally, the values of $F_{j}, f_{j}$ and $\beta$ are obtained using Eqs. (A.19)-(A.21). 


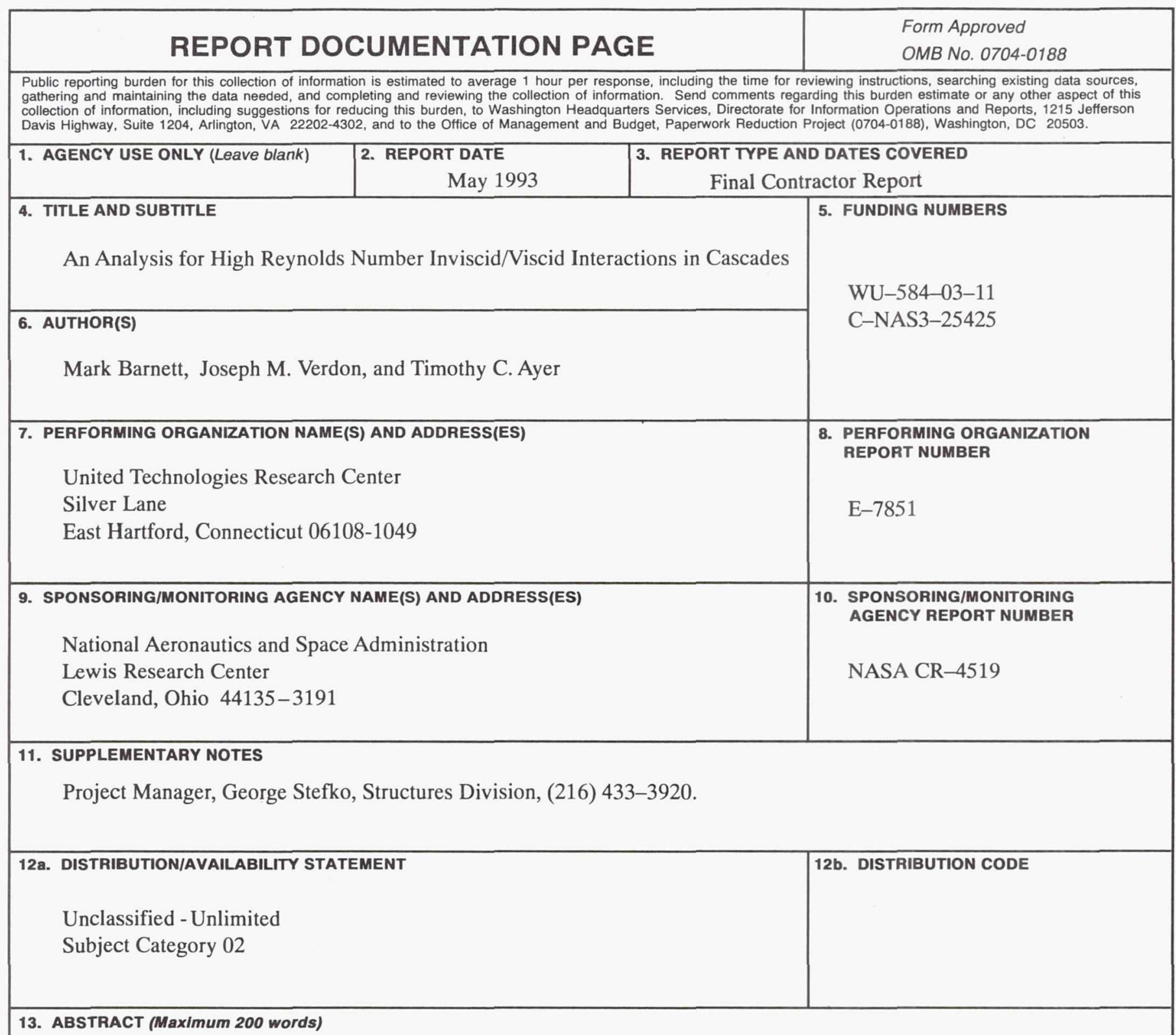

13. ABSTRACT (Maximum 200 words)

An efficient steady analysis for predicting strong inviscid/viscid interaction phenomena such as viscous-layer separation, shock/boundary-layer interaction and trailing-edge/near-wake interaction in turbomachinery blade passages is needed as part of a comprehensive analytical blade design prediction system. Such an analysis is described in the present report. It uses an inviscid/viscid interaction approach, in which the flow in the outer inviscid region is assumed to be potential, and that in the inner or viscous-layer region is governed by Prandtl's equations. The inviscid solution is determined using an implicit, least-squares, finite-difference approximation, the viscous-layer solution using an inverse, finite-difference, space-marching method which is applied along the blade surfaces and wake streamlines. The inviscid and viscid solutions are coupled using a semi-inverse global iteration procedure, which permits the prediction of boundary-layer separation and other strong-interaction phenomena. Results are presented for three cascades, with a range of inlet flow conditions considered for one of them, including conditions leading to large-scale flow separations. Comparisons with Navier-Stokes solutions and experimental data are also given.

\begin{tabular}{|c|c|c|c|}
\hline 14. SUBJECT TERMS & & & $\begin{array}{c}\text { 15. NUMBER OF PAGES } \\
50\end{array}$ \\
\hline \multicolumn{3}{|c|}{ Unsteady aerodynamics; Cascades } & 16. PRICE CODE \\
\hline $\begin{array}{l}\text { 17. SECURITY CLASSIFICATION } \\
\text { OF REPORT }\end{array}$ & $\begin{array}{l}\text { 18. SECURITY CLASSIFICATION } \\
\text { OF THIS PAGE }\end{array}$ & $\begin{array}{l}\text { 19. SECURITY CLASSIFICATION } \\
\text { OF ABSTRACT }\end{array}$ & 20. LIMITATION OF ABSTRACT \\
\hline Unclassified & Unclassified & Unclassified & \\
\hline
\end{tabular}

